\title{
Multiple Hydrogen Bonding for the Non-Covalent Attachment of Ionic Functionality in Triblock Copolymers
}

\author{
Brian D. Mather, Margaux B. Baker, Frederick L. Beyer, Matthew D. Green, \\ Michael A.G. Berg, Timothy E. Long
}

\section{Supporting Information}

\section{Materials}

$n$-Butyl acrylate (99\%) was purchased from Aldrich and purified using an alumina column and subsequent vacuum distillation from calcium hydride. Diethyl-meso-2,5-dibromoadipate (98\%), copper (I) bromide (99.999\%), $N, N, N$ ', $N$ ', $N$ '-pentamethyldiethylenetriamine (PMDETA, 98\%), trioctylphosphine $(90 \%)$ and 6-chloromethyluracil (98\%) were purchased from Aldrich and used without further purification. Copper powder $(45 \mu \mathrm{m}, 99 \%)$ was purchased from Acros and used as received. DEPN nitroxide, ${ }^{1}$ Styryl-DEPN, ${ }^{2}$ and 9-vinylbenzyl adenine ${ }^{3}$ were synthesized according to the previous literature.

\section{Synthesis of 6-(trioctylphosphonium methyl)uracil chloride $\left(\mathrm{UP}^{+}\right)$}

6-chloromethyluracil $(1.001 \mathrm{~g}, 6.232 \mathrm{mmol})$ and trioctylphosphine $(3.25 \mathrm{~mL}, 2.70 \mathrm{~g}$, $7.29 \mathrm{mmol}$ ) were charged to a $100 \mathrm{~mL}$ round-bottomed flask containing a magnetic stirbar. Ethanol $(40 \mathrm{~mL})$ was added and a condenser was fitted to the flask. The reactions was heated to reflux using an external oil bath for $24 \mathrm{~h}$. Excess solvent was removed using rotary evaporation. The resulting waxy solid was washed with diethyl ether and was dried overnight at room temperature under high vacuum. $\mathrm{UP}^{+}$was obtained as a light brown solid (m.p. $179-181{ }^{\circ} \mathrm{C}$ ) in $44 \%$ yield. ${ }^{1} \mathrm{H}$ NMR (400 MHz, DMSO-d $\left._{6}, 25^{\circ} \mathrm{C}\right) \delta(\mathrm{ppm}): 0.86(\mathrm{t}, 9 \mathrm{H}, J=6.8 \mathrm{~Hz}), 1.26(\mathrm{~m}, 24 \mathrm{H}), 1.37(\mathrm{~m}, 6 \mathrm{H}), 1.49$ $(\mathrm{m}, 6 \mathrm{H}), 2.37(\mathrm{~m}, 6 \mathrm{H}), 3.77(\mathrm{~d}, 2 \mathrm{H}, J=15 \mathrm{~Hz}), 5.55(\mathrm{~m}, 1 \mathrm{H}), 11.15(\mathrm{~s}, 1 \mathrm{H}), 11.47(\mathrm{~s}$, 1H). ${ }^{13} \mathrm{C}$ NMR (101 MHz, DMSO-d $\left.6,25{ }^{\circ} \mathrm{C}\right) \delta(\mathrm{ppm}): 13.96,17.61,18.06,20.52$, 22.08, 28.27, 28.8, 30.06, 31.22, 102.08, 145.18, 150.95, 163.37. ${ }^{31} \mathrm{P}$ NMR (162 MHz, $\left.\mathrm{DMSO}_{6}, 25{ }^{\circ} \mathrm{C}\right) \delta(\mathrm{ppm}): 33.68 \mathrm{ppm}$. FAB MS: $\mathrm{m} / z=495.4056[\mathrm{M}-\mathrm{Cl}]^{+}$ (experimental), $\mathrm{m} / z=495.41$ (theoretical).

\section{Synthesis of $\mathrm{DEPN}_{2}$ Difunctional Alkoxyamine Initiator}

DEPN nitroxide (2.0 g, $6.8 \mathrm{mmol})$ and diethyl-meso-2,5-dibromoadipate (1.36 g, 3.8 mmol) were charged to a one-necked $100-\mathrm{mL}$ round-bottomed flask containing a magnetic stirbar. Dichloromethane $(30 \mathrm{~mL})$ was added and the solution was degassed with three freeze-pump-thaw cycles. In a second $100-\mathrm{mL}$ round-bottomed flask, PMDETA (2.34 g, $13.6 \mathrm{mmol}$ ) was dissolved in $20 \mathrm{~mL} \mathrm{CH}_{2} \mathrm{Cl}_{2}$ and subjected to the same degassing. In a third $100-\mathrm{mL}$ round-bottomed flask containing a stirbar, $\mathrm{CuBr}$ $(0.78 \mathrm{~g}, 5.47 \mathrm{mmol})$ and $\mathrm{Cu}$ powder $(0.35 \mathrm{~g}, 5.47 \mathrm{mmol})$ were purged with nitrogen gas. After degassing, the liquid reagents were cannulated into the flask containing the copper powder and $\mathrm{CuBr}$. The reaction was stirred for $24 \mathrm{~h}$ at $25{ }^{\circ} \mathrm{C}$ under nitrogen, diluted with $\mathrm{CH}_{2} \mathrm{Cl}_{2}(200 \mathrm{~mL})$, and washed repeatedly with water $(10 \mathrm{x} 100 \mathrm{~mL})$, to 
remove copper compounds. The organic layer was then dried over sodium sulfate and evaporated. The product was separated on silica, eluting with ethyl acetate : hexane (1:1). DEPN 2 was isolated as a mixture of diastereomers. Purified yield $=49 \%$. ${ }^{1} \mathrm{H}$ NMR (400 MHz, $\mathrm{CDCl}_{3}, 25^{\circ} \mathrm{C}$ ) $\delta$ (ppm): 1.0-1.2 (s, tBu, 36H), 1.2-1.4 (m, ester $\mathrm{CH}_{3}$, 18H), 1.6-2.4 (br, linker $\left.\mathrm{CH}_{2}, 4 \mathrm{H}\right), 3.1-3.6$ (m, DEPN CH, 2H), 3.9-4.2 (m, $\left.\mathrm{CH}_{3}, 12 \mathrm{H}\right)$, 4.4-4.5 (m, COCH, 2H). $\left.{ }^{13} \mathrm{C} \mathrm{NMR} \mathrm{(101} \mathrm{MHz,} \mathrm{CDCl}_{3}, 25^{\circ} \mathrm{C}\right) \delta(\mathrm{ppm}): 172.74,171.46$, $86.74,86.31,81.87,81.52,77.20,76.85,75.31,70.27,69.99,68.90,68.62,62.30$, $62.23,62.03,61.96,61.66,61.57,60.62,60.40,60.32,60.27,60.22,58.89,58.82$, $35.64,35.58,35.49,35.21,35.16,35.09,34.48,30.18,30.12$, 29.93, 29.89, 29.44, $29.38,28.12,27.95,27.89,27.56,27.53,26.96,26.67,26.52,26.46,16.47,16.41$, $16.35,16.32,16.14,16.08,14.03,13.98,13.90 .{ }^{31} \mathrm{P} \mathrm{NMR}\left(162 \mathrm{MHz}, \mathrm{CDCl}_{3}, 25{ }^{\circ} \mathrm{C}\right) \delta$ (ppm): 25.82, 25.63, 25.59, 25.20, 25.00, 24.91. FAB MS: $\mathrm{m} / z=789.4842[\mathrm{M}+\mathrm{H}]^{+}$ experimental, $m / z=789.48$ theoretical. No peak for the monofunctional initiator was observed $(\mathrm{m} / \mathrm{z}=574.5)$.

Meso diastereomer of $\mathrm{DEPN}_{2}$ : The meso diastereomer was isolated from the partly crystalline first fractions obtained during chromatographic purification of $\mathrm{DEPN}_{2}$. Further separation was achieved via washing the crystals with hexane. m.p. = 139.5-141.5 ${ }^{\circ} \mathrm{C} .{ }^{1} \mathrm{H}$ NMR (400 MHz, $\left.\mathrm{CDCl}_{3}, 25^{\circ} \mathrm{C}\right) \delta(\mathrm{ppm}): 1.03$ (s, $N$-tBu, $\left.18 \mathrm{H}\right)$, 1.06 (s, $C$-tBu, $18 \mathrm{H}$ ), 1.24 (m, ester $\mathrm{CH}_{3}, 18 \mathrm{H}$ ), 1.55-2.40 (br, linker $\mathrm{CH}_{2}, 4 \mathrm{H}$ ), 3.18 (d, $J=25 \mathrm{~Hz}$, DEPN CH, 2H), 3.85-4.20 (m, $\left.\mathrm{OCH}_{2}, 12 \mathrm{H}\right), 4.25-4.46(\mathrm{br}, \mathrm{OCH}, 2 \mathrm{H}) .{ }^{13} \mathrm{C}$ NMR (101 MHz, $\left.\mathrm{CDCl}_{3}, 25^{\circ} \mathrm{C}\right) \delta(\mathrm{ppm}): 172.89,86.65,69.71(\mathrm{~d}, J=138 \mathrm{~Hz}), \quad 61.80$ $(\mathrm{d}, J=6.1 \mathrm{~Hz}), 61.05(\mathrm{~d}, J=95 \mathrm{~Hz}), 58.69$ (d, J = $7 \mathrm{~Hz}), 35.59$ (d, $J=5.4 \mathrm{~Hz}), 29.31$ (d, $J=6.2 \mathrm{~Hz}), 28.57,27.94,16.31\left(\mathrm{dd}, J_{1}=28.4 \mathrm{~Hz}, J_{2}=6.2 \mathrm{~Hz}\right), 13.95 .{ }^{31} \mathrm{P}$ NMR $(162$ $\left.\mathrm{MHz}, \mathrm{CDCl}_{3}, 25^{\circ} \mathrm{C}\right) \delta(\mathrm{ppm}): 25.67$.

\section{Polymerization of n-Butyl Acrylate from $\mathrm{DEPN}_{2}$ Nitroxide}

$\mathrm{DEPN}_{2}$ alkoxyamine $(212 \mathrm{mg}, 0.269 \mathrm{mmol})$ and DEPN nitroxide (31 mg, 0.106 mmol) were weighed into a $100-\mathrm{mL}$ round-bottomed flask containing a magnetic stirbar. The flask was sealed with a three-way joint allowing syringing of reagents and application of vacuum and nitrogen. The flask was evacuated to 60 mtorr and refilled with high-purity nitrogen three times. Purified $n$-butyl acrylate $(30 \mathrm{~mL}, 209 \mathrm{mmol}$ ) was added via syringe and the mixture was degassed with repeated freeze-pump-thaw cycles. Finally, the flask was immersed in an oil bath at $122{ }^{\circ} \mathrm{C}$ for $3 \mathrm{~h}$. After the polymerization, residual monomer was removed with high vacuum stripping. Yield = $5.82 \mathrm{~g}$ polymer $($ Conversion $=22 \%)$. SEC analysis revealed molecular weight data $M_{n}$ $=16,500, \mathrm{M}_{\mathrm{w}} / \mathrm{M}_{\mathrm{n}}=1.24$.

\section{Synthesis of Adenine Containing Block Copolymer}

$\operatorname{Poly}\left(n\right.$-butyl acrylate) homopolymer (4.83 g, $\left.\mathrm{M}_{\mathrm{n}}=16,500\right)$ and 9-vinylbenzyl adenine $^{13}$ (9-VBA, $0.990 \mathrm{~g}, 3.94 \mathrm{mmol}$ ) were added to a 100-mL round-bottomed flask with a magnetic stirbar. The flask was sealed with a three-way joint and evacuated to 60 mtorr and refilled with high-purity nitrogen three times. DMF $(15 \mathrm{~mL})$ was then syringed into the flask and the mixture stirred until homogeneous and then degassed repeatedly with freeze-pump-thaw cycles. The flask was immersed in an oil bath at 120 
${ }^{\circ} \mathrm{C}$ for $5 \mathrm{~h}$. After the polymerization, the polymer was isolated via precipitation twice into methanol. ${ }^{1} \mathrm{H}$ NMR $\left(400 \mathrm{MHz}, \mathrm{DMSO}-\mathrm{d}_{6}: \mathrm{CDCl}_{3}, 1: 1,25{ }^{\circ} \mathrm{C}\right)$ revealed block molecular weights $1.5 \mathrm{~K}-16.5 \mathrm{~K}-1.5 \mathrm{~K}$. The final product was dried for $24 \mathrm{~h}$ under high vacuum at room temperature and stored in a dessicator. The adenine triblock copolymer formed a clear, elastomeric polymer.

Blend of adenine functional block copolymer with $\mathrm{UP}^{+}$(1:1 molar A:U ratio) Adenine containing block copolymer ( $1.00 \mathrm{~g}, 0.612 \mathrm{mmol}$ adenine) and $\mathrm{UP}^{+}(338 \mathrm{mg}$, $0.637 \mathrm{mmol}$ ) were blended in $15 \mathrm{~mL}$ chloroform, and cast in a Teflon mold and dried over $24 \mathrm{~h}$. Homogeneity was maintained upon drying.

\section{Evidence of Uracil Phosphonium Salt - 9-VBA Hydrogen Bonding Association from ${ }^{1} \mathrm{H} N M R$}

9-VBA (14.2 mg, $0.056 \mathrm{mmol})$ was dissolved in $\mathrm{CDCl}_{3}(2.44 \mathrm{~g}, 1.62 \mathrm{mM})$ and characterized via ${ }^{1} \mathrm{H}$ NMR spectroscopy. The position of the adenine $\mathrm{NH}_{2}$ peak was noted at $5.74 \mathrm{ppm}$. $\mathrm{UP}^{+}$was added incrementally with proton NMR spectra collected between additions. The $\mathrm{NH}_{2}$ peak was found to shift monotonically downfield to 5.92 ppm after addition of 0.29 equiv of $\mathrm{UP}^{+}$.

For the adenine functional block copolymer, difficulty in observing the adenine functional blocks in $\mathrm{CDCl}_{3}$ prevented the analogous experiment.

\section{Characterization}

Size exclusion chromatography (SEC) was performed at $40{ }^{\circ} \mathrm{C}$ in HPLC grade tetrahydrofuran at $1 \mathrm{~mL} / \mathrm{min}$ using a Waters size-exclusion chromatographer equipped with an autosampler, 3 in-line $5 \mu \mathrm{m}$ PLgel MIXED-C columns. Detectors included a Waters 410 differential refractive index (DRI) detector operating at $880 \mathrm{~nm}$, and a Wyatt Technologies miniDAWN multiangle $690 \mathrm{~nm}$ laser light scattering (MALLS) detector, calibrated with PS standards. NMR spectroscopic data was collected on a Varian $400 \mathrm{MHz}$ spectrometer at $25{ }^{\circ} \mathrm{C}$. A Veeco MultiMode ${ }^{\mathrm{TM}}$ scanning probe microscope was used for tapping-mode AFM. Polymer films were solution cast from chloroform on silicon. Samples were imaged at a set-point ratio of 0.6-0.7. Veeco's Nanosensor silicon tips having spring constants of 10-130 N/m were utilized for imaging. Solution rheology measurements were conducted on a TA Instruments G2 Rheometer in concentric cylinder geometry. X-ray data was collected on an Oxford Diffraction Xcalibur ${ }^{\mathrm{TM}}$ diffractometer equipped with a Sapphire ${ }^{\mathrm{TM}} 3$ CCD detector. The data collection routine, unit cell refinement, and data processing were carried out with the program CrysAlis(v1.171, Oxford Diffraction: Wroclaw, Poland, 2004).

\section{Small-angle X-ray Scattering (SAXS) Measurements}

$\mathrm{Cu}_{\mathrm{K} \alpha \square} \mathrm{X}$-ray radiation was generated using a Rigaku Ultrax 18 rotating anode X-ray generator operated at $45 \mathrm{kV}$ and $100 \mathrm{~mA}$. A nickel filter was used to eliminate all wavelengths but the $\mathrm{Cu}_{\mathrm{K} \alpha}$ doublet, with an average wavelength of $\lambda=1.542 \AA$. The exact beam center and the sample-to-detector distance of approximately $1.5 \mathrm{~m}$ were 
calibrated using silver behenate. The $3 \mathrm{~m}$ camera uses 3-pinhole collimation (300, 200 and $600 \mu \mathrm{m}$ ), and a sample-to-detector distance of approximately $1.5 \mathrm{~m}$. Two-dimensional data sets were collected using a Molecular Metrology 2D multi-wire area detector. The data were corrected for detector noise, sample absorption, and background scattering, followed by azimuthal averaging to obtain intensity as a function of the scattering vector, $\mathrm{I}(q)$, where $q=4 \pi \cdot \sin (\theta) / \lambda$ and $2 \theta$ is the scattering angle. The data were then placed on an absolute scale using a type 2 glassy carbon sample $1.07 \mathrm{~mm}$ thick, previously calibrated at the Advanced Photon Source in the Argonne National Laboratory, as a secondary standard. All data processing and analysis were done using Wavemetrics IGOR Pro 5.04 software and IGOR procedures written by Dr. Jan Ilavsky of Argonne National Laboratory.

\section{References}

(1) Grimaldi, S,; Finet, J.-P. Le Moigne, F; Zeghdaoui, A.; Tordo, P.; Benoitm D.; Fontanille, M.; Gnanou, Y. Macromolecules 2000, 33, 1141-1147.

(2) Diaz, T.; Fischer, A.; Jonquieres, A.; Brembilla, A.; Lochon, P., Macromolecules 2003, 36, 2235-2241.

(3) Srivatsan, S. G.; Parvez, M.; Verma, S., Chem. Eur. J. 2002, 8, 5184-5191. 
Table 1. Crystal data and structure refinement for $\mathrm{DEPN}_{2}$.

Empirical formula $\quad \mathrm{C}_{36} \mathrm{H}_{74} \mathrm{~N}_{2} \mathrm{O}_{12} \mathrm{P}_{2}$

Formula weight $\quad 788.91$

Temperature $\quad$ 100(2) K

Wavelength $\quad 0.71073 \AA$

Crystal system monoclinic

Space group $\quad \mathrm{C} 2 / \mathrm{c}$

Unit cell dimensions $\mathrm{a}=18.903(3) \AA$.

$$
\begin{aligned}
& \mathrm{b}=14.8584(14) \AA \quad \beta=117.94(2)^{\circ} . \\
& \mathrm{c}=17.677(3) \AA .
\end{aligned}
$$

Volume 4386.2(11) $\AA^{3}$

Z 4

Density (calculated) $\quad 1.195 \mathrm{Mg} / \mathrm{m}^{3}$

Absorption coefficient $\quad 0.156 \mathrm{~mm}^{-1}$

$\mathrm{F}(000) \quad 1720$

Crystal size $\quad 0.18 \times 0.29 \times 0.38 \mathrm{~mm}^{3}$

Theta range for data collection 3.62 to $25.07^{\circ}$.

Index ranges $\quad-22<=\mathrm{h}<=22,-17<=\mathrm{k}<=17,-21<=\mathrm{l}<=21$

Reflections collected 29231

Independent reflections 3896

Completeness to theta $=25.00^{\circ} \quad 99.7 \%$

Refinement method Full-matrix least-squares on $\mathrm{F}^{2}$

Data / restraints / parameters 3896 / 0 / 244

Goodness-of-fit on $\mathrm{F}^{2} \quad 1.030$

Final R indices [I $>2 \operatorname{sigma}(\mathrm{I})] \mathrm{R} 1=0.0433, \mathrm{wR} 2=0.1171$

$\mathrm{R}$ indices (all data) $\quad \mathrm{R} 1=0.0627, \mathrm{wR} 2=0.1224$

Largest diff. peak and hole $\quad 0.559$ and -0.371 e. $\AA^{-3}$ 
Table 2. Atomic coordinates ( $\mathrm{x} 10^{4}$ ) and equivalent isotropic displacement parameters $\left(\AA^{2} \times 10^{3}\right)$ for $\mathrm{DEPN}_{2}$. U(eq) is defined as one third of the trace of the orthogonalized $\mathrm{U}^{\mathrm{ij}}$ tensor.

\begin{tabular}{|c|c|c|c|}
\hline & $\mathrm{x}$ & $\mathrm{y}$ & $\mathrm{U}(\mathrm{eq})$ \\
\hline $\mathrm{C}(8)$ & $3796(2)$ & $7483(2)-1977(2$ & $44(1)$ \\
\hline $\mathrm{C}(9)$ & $1638(1)$ & $3946(2) 2103(1)$ & $38(1)$ \\
\hline $\mathrm{C}(10)$ & $1275(2)$ & $3065(2) 2134(2)$ & $51(1)$ \\
\hline $\mathrm{C}(11)$ & $3533(2)$ & $5544(2) 2290(2)$ & $65(1)$ \\
\hline$C(12)$ & $4307(2)$ & $5235(2) 2997(2)$ & $77(1)$ \\
\hline $\mathrm{C}(1)$ & $3633(1)$ & $6631(1)-148(1)$ & $26(1)$ \\
\hline $\mathrm{C}(2)$ & $2879(1)$ & $6322(1)-122(1)$ & $24(1)$ \\
\hline $\mathrm{C}(3)$ & $2616(1)$ & $7075(1) 274(1)$ & $26(1)$ \\
\hline $\mathrm{C}(4)$ & $2035(1)$ & $4417(1) 113(1)$ & $25(1)$ \\
\hline$C(5)$ & 1159(1) & $4580(1)-626(1)$ & $34(1)$ \\
\hline$C(6)$ & $3295(1)$ & $4083(1)-5(1)$ & $32(1)$ \\
\hline $\mathrm{C}(7)$ & 4113(1) & $7260(2)-1050(1$ & ) $35(1)$ \\
\hline $\mathrm{C}(51)$ & $941(1)$ & $5576(2)-834(2)$ & $41(1)$ \\
\hline$C(52)$ & $546(1)$ & $4143(2)-401(2)$ & $46(1)$ \\
\hline $\mathrm{C}(53)$ & $1080(2)$ & $4121(2)-1438(2$ & $52(1)$ \\
\hline$C(61)$ & $3747(1)$ & $3690(1) 898(1)$ & $35(1)$ \\
\hline$C(62)$ & $3878(2)$ & $4574(2)-234(2)$ & $47(1)$ \\
\hline $\mathrm{C}(63)$ & 2913(2) & $3323(2)-655(2)$ & $42(1)$ \\
\hline $\mathrm{N}(1)$ & $2644(1)$ & $4742(1)-129(1)$ & $25(1)$ \\
\hline $\mathrm{O}(1)$ & $3471(1)$ & $6833(1)-951(1)$ & $27(1)$ \\
\hline $\mathrm{O}(2)$ & $4279(1)$ & $6731(1) 469(1)$ & $36(1)$ \\
\hline $\mathrm{O}(3)$ & $3019(1)$ & $5531(1) 398(1)$ & $24(1)$ \\
\hline $\mathrm{O}(4)$ & $3066(1)$ & $4755(1) 1854(1)$ & $32(1)$ \\
\hline $\mathrm{O}(5)$ & 1761(1) & $5636(1) 1175(1)$ & $39(1)$ \\
\hline $\mathrm{O}(6)$ & $1824(1)$ & $3915(1)$ 1393(1) & $32(1)$ \\
\hline $\mathrm{P}(1)$ & $2159(1)$ & $4795(1) 1162(1)$ & $27(1)$ \\
\hline
\end{tabular}

Table 3. Bond lengths $[\AA]$ and angles $\left[{ }^{\circ}\right]$ for $\mathrm{DEPN}_{2}$.

\begin{tabular}{lc}
\hline $\mathrm{C}(8)-\mathrm{C}(7)$ & $1.495(3)$ \\
$\mathrm{C}(8)-\mathrm{H}(8 \mathrm{~A})$ & 0.9800 \\
$\mathrm{C}(8)-\mathrm{H}(8 \mathrm{~B})$ & 0.9800 \\
$\mathrm{C}(8)-\mathrm{H}(8 \mathrm{C})$ & 0.9800 \\
$\mathrm{C}(9)-\mathrm{O}(6)$ & $1.455(2)$ \\
$\mathrm{C}(9)-\mathrm{C}(10)$ & $1.490(3)$ \\
$\mathrm{C}(9)-\mathrm{H}(9 \mathrm{~A})$ & 0.9900 \\
$\mathrm{C}(9)-\mathrm{H}(9 \mathrm{~B})$ & 0.9900
\end{tabular}




\begin{tabular}{|c|c|}
\hline $\mathrm{C}(10)-\mathrm{H}(10 \mathrm{~A})$ & 0.9800 \\
\hline $\mathrm{C}(10)-\mathrm{H}(10 \mathrm{~B})$ & 0.9800 \\
\hline $\mathrm{C}(10)-\mathrm{H}(10 \mathrm{C})$ & 0.9800 \\
\hline $\mathrm{C}(11)-\mathrm{O}(4)$ & $1.452(3)$ \\
\hline$C(11)-C(12)$ & $1.483(4)$ \\
\hline $\mathrm{C}(11)-\mathrm{H}(11 \mathrm{~A})$ & 0.9900 \\
\hline $\mathrm{C}(11)-\mathrm{H}(11 \mathrm{~B})$ & 0.9900 \\
\hline $\mathrm{C}(12)-\mathrm{H}(12 \mathrm{~A})$ & 0.9800 \\
\hline $\mathrm{C}(12)-\mathrm{H}(12 \mathrm{~B})$ & 0.9800 \\
\hline $\mathrm{C}(12)-\mathrm{H}(12 \mathrm{C})$ & 0.9800 \\
\hline $\mathrm{C}(1)-\mathrm{O}(2)$ & $1.205(2)$ \\
\hline $\mathrm{C}(1)-\mathrm{O}(1)$ & $1.338(2)$ \\
\hline $\mathrm{C}(1)-\mathrm{C}(2)$ & $1.518(3)$ \\
\hline $\mathrm{C}(2)-\mathrm{O}(3)$ & $1.439(2)$ \\
\hline$C(2)-C(3)$ & $1.521(3)$ \\
\hline $\mathrm{C}(2)-\mathrm{H}(2)$ & 1.0000 \\
\hline$C(3)-C(3) \# 1$ & $1.524(4)$ \\
\hline $\mathrm{C}(3)-\mathrm{H}(3 \mathrm{~A})$ & 0.9900 \\
\hline $\mathrm{C}(3)-\mathrm{H}(3 \mathrm{~B})$ & 0.9900 \\
\hline $\mathrm{C}(4)-\mathrm{N}(1)$ & $1.484(2)$ \\
\hline$C(4)-C(5)$ & $1.575(3)$ \\
\hline $\mathrm{C}(4)-\mathrm{P}(1)$ & $1.844(2)$ \\
\hline $\mathrm{C}(4)-\mathrm{H}(4)$ & 1.0000 \\
\hline$C(5)-C(53)$ & $1.532(3)$ \\
\hline$C(5)-C(51)$ & $1.533(3)$ \\
\hline$C(5)-C(52)$ & $1.534(3)$ \\
\hline $\mathrm{C}(6)-\mathrm{N}(1)$ & $1.506(3)$ \\
\hline$C(6)-C(62)$ & $1.526(3)$ \\
\hline$C(6)-C(63)$ & $1.529(3)$ \\
\hline$C(6)-C(61)$ & $1.530(3)$ \\
\hline $\mathrm{C}(7)-\mathrm{O}(1)$ & $1.451(2)$ \\
\hline $\mathrm{C}(7)-\mathrm{H}(7 \mathrm{~A})$ & 0.9900 \\
\hline $\mathrm{C}(7)-\mathrm{H}(7 \mathrm{~B})$ & 0.9900 \\
\hline $\mathrm{C}(51)-\mathrm{H}(51 \mathrm{~A})$ & 0.9800 \\
\hline $\mathrm{C}(51)-\mathrm{H}(51 \mathrm{~B})$ & 0.9800 \\
\hline $\mathrm{C}(51)-\mathrm{H}(51 \mathrm{C})$ & 0.9800 \\
\hline $\mathrm{C}(52)-\mathrm{H}(52 \mathrm{~A})$ & 0.9800 \\
\hline $\mathrm{C}(52)-\mathrm{H}(52 \mathrm{~B})$ & 0.9800 \\
\hline $\mathrm{C}(52)-\mathrm{H}(52 \mathrm{C})$ & 0.9800 \\
\hline $\mathrm{C}(53)-\mathrm{H}(53 \mathrm{~A})$ & 0.9800 \\
\hline $\mathrm{C}(53)-\mathrm{H}(53 \mathrm{~B})$ & 0.9800 \\
\hline $\mathrm{C}(53)-\mathrm{H}(53 \mathrm{C})$ & 0.9800 \\
\hline $\mathrm{C}(61)-\mathrm{H}(61 \mathrm{~A})$ & 0.9800 \\
\hline $\mathrm{C}(61)-\mathrm{H}(61 \mathrm{~B})$ & 0.9800 \\
\hline
\end{tabular}




\begin{tabular}{|c|c|}
\hline $\mathrm{C}(61)-\mathrm{H}(61 \mathrm{C})$ & 0.9800 \\
\hline $\mathrm{C}(62)-\mathrm{H}(62 \mathrm{~A})$ & 0.9800 \\
\hline $\mathrm{C}(62)-\mathrm{H}(62 \mathrm{~B})$ & 0.9800 \\
\hline $\mathrm{C}(62)-\mathrm{H}(62 \mathrm{C})$ & 0.9800 \\
\hline $\mathrm{C}(63)-\mathrm{H}(63 \mathrm{~A})$ & 0.9800 \\
\hline $\mathrm{C}(63)-\mathrm{H}(63 \mathrm{~B})$ & 0.9800 \\
\hline $\mathrm{C}(63)-\mathrm{H}(63 \mathrm{C})$ & 0.9800 \\
\hline $\mathrm{N}(1)-\mathrm{O}(3)$ & $1.459(2)$ \\
\hline $\mathrm{O}(4)-\mathrm{P}(1)$ & $1.5729(16)$ \\
\hline $\mathrm{O}(5)-\mathrm{P}(1)$ & $1.4650(15)$ \\
\hline $\mathrm{O}(6)-\mathrm{P}(1)$ & $1.5867(14)$ \\
\hline $\mathrm{C}(7)-\mathrm{C}(8)-\mathrm{H}(8 \mathrm{~A})$ & 109.5 \\
\hline $\mathrm{C}(7)-\mathrm{C}(8)-\mathrm{H}(8 \mathrm{~B})$ & 109.5 \\
\hline $\mathrm{H}(8 \mathrm{~A})-\mathrm{C}(8)-\mathrm{H}(8 \mathrm{~B})$ & 109.5 \\
\hline $\mathrm{C}(7)-\mathrm{C}(8)-\mathrm{H}(8 \mathrm{C})$ & 109.5 \\
\hline $\mathrm{H}(8 \mathrm{~A})-\mathrm{C}(8)-\mathrm{H}(8 \mathrm{C})$ & 109.5 \\
\hline $\mathrm{H}(8 \mathrm{~B})-\mathrm{C}(8)-\mathrm{H}(8 \mathrm{C})$ & 109.5 \\
\hline $\mathrm{O}(6)-\mathrm{C}(9)-\mathrm{C}(10)$ & $107.83(18)$ \\
\hline $\mathrm{O}(6)-\mathrm{C}(9)-\mathrm{H}(9 \mathrm{~A})$ & 110.1 \\
\hline $\mathrm{C}(10)-\mathrm{C}(9)-\mathrm{H}(9 \mathrm{~A})$ & 110.1 \\
\hline $\mathrm{O}(6)-\mathrm{C}(9)-\mathrm{H}(9 \mathrm{~B})$ & 110.1 \\
\hline $\mathrm{C}(10)-\mathrm{C}(9)-\mathrm{H}(9 \mathrm{~B})$ & 110.1 \\
\hline $\mathrm{H}(9 \mathrm{~A})-\mathrm{C}(9)-\mathrm{H}(9 \mathrm{~B})$ & 108.5 \\
\hline $\mathrm{C}(9)-\mathrm{C}(10)-\mathrm{H}(10 \mathrm{~A})$ & 109.5 \\
\hline $\mathrm{C}(9)-\mathrm{C}(10)-\mathrm{H}(10 \mathrm{~B})$ & 109.5 \\
\hline $\mathrm{H}(10 \mathrm{~A})-\mathrm{C}(10)-\mathrm{H}(10 \mathrm{~B})$ & 109.5 \\
\hline $\mathrm{C}(9)-\mathrm{C}(10)-\mathrm{H}(10 \mathrm{C})$ & 109.5 \\
\hline $\mathrm{H}(10 \mathrm{~A})-\mathrm{C}(10)-\mathrm{H}(10 \mathrm{C})$ & 109.5 \\
\hline $\mathrm{H}(10 \mathrm{~B})-\mathrm{C}(10)-\mathrm{H}(10 \mathrm{C})$ & 109.5 \\
\hline $\mathrm{O}(4)-\mathrm{C}(11)-\mathrm{C}(12)$ & $108.2(2)$ \\
\hline $\mathrm{O}(4)-\mathrm{C}(11)-\mathrm{H}(11 \mathrm{~A})$ & 110.1 \\
\hline $\mathrm{C}(12)-\mathrm{C}(11)-\mathrm{H}(11 \mathrm{~A})$ & 110.1 \\
\hline $\mathrm{O}(4)-\mathrm{C}(11)-\mathrm{H}(11 \mathrm{~B})$ & 110.1 \\
\hline $\mathrm{C}(12)-\mathrm{C}(11)-\mathrm{H}(11 \mathrm{~B})$ & 110.1 \\
\hline $\mathrm{H}(11 \mathrm{~A})-\mathrm{C}(11)-\mathrm{H}(11 \mathrm{~B})$ & 108.4 \\
\hline $\mathrm{C}(11)-\mathrm{C}(12)-\mathrm{H}(12 \mathrm{~A})$ & 109.5 \\
\hline $\mathrm{C}(11)-\mathrm{C}(12)-\mathrm{H}(12 \mathrm{~B})$ & 109.5 \\
\hline $\mathrm{H}(12 \mathrm{~A})-\mathrm{C}(12)-\mathrm{H}(12 \mathrm{~B})$ & 109.5 \\
\hline $\mathrm{C}(11)-\mathrm{C}(12)-\mathrm{H}(12 \mathrm{C})$ & 109.5 \\
\hline $\mathrm{H}(12 \mathrm{~A})-\mathrm{C}(12)-\mathrm{H}(12 \mathrm{C})$ & 109.5 \\
\hline $\mathrm{H}(12 \mathrm{~B})-\mathrm{C}(12)-\mathrm{H}(12 \mathrm{C})$ & 109.5 \\
\hline $\mathrm{O}(2)-\mathrm{C}(1)-\mathrm{O}(1)$ & $124.26(18)$ \\
\hline $\mathrm{O}(2)-\mathrm{C}(1)-\mathrm{C}(2)$ & $125.23(18)$ \\
\hline $\mathrm{O}(1)-\mathrm{C}(1)-\mathrm{C}(2)$ & $110.38(16)$ \\
\hline
\end{tabular}




$\begin{array}{ll}\mathrm{O}(3)-\mathrm{C}(2)-\mathrm{C}(1) & 111.83(15) \\ \mathrm{O}(3)-\mathrm{C}(2)-\mathrm{C}(3) & 107.99(15) \\ \mathrm{C}(1)-\mathrm{C}(2)-\mathrm{C}(3) & 107.82(16) \\ \mathrm{O}(3)-\mathrm{C}(2)-\mathrm{H}(2) & 109.7 \\ \mathrm{C}(1)-\mathrm{C}(2)-\mathrm{H}(2) & 109.7 \\ \mathrm{C}(3)-\mathrm{C}(2)-\mathrm{H}(2) & 109.7 \\ \mathrm{C}(2)-\mathrm{C}(3)-\mathrm{C}(3) \# 1 & 112.1(2) \\ \mathrm{C}(2)-\mathrm{C}(3)-\mathrm{H}(3 \mathrm{~A}) & 109.2 \\ \mathrm{C}(3)+11-\mathrm{C}(3)-\mathrm{H}(3 \mathrm{~A}) & 109.2 \\ \mathrm{C}(2)-\mathrm{C}(3)-\mathrm{H}(3 \mathrm{~B}) & 109.2 \\ \mathrm{C}(3)+11-\mathrm{C}(3)-\mathrm{H}(3 \mathrm{~B}) & 109.2 \\ \mathrm{H}(3 \mathrm{~A})-\mathrm{C}(3)-\mathrm{H}(3 \mathrm{~B}) & 107.9 \\ \mathrm{~N}(1)-\mathrm{C}(4)-\mathrm{C}(5) & 111.52(16) \\ \mathrm{N}(1)-\mathrm{C}(4)-\mathrm{P}(1) & 116.12(13) \\ \mathrm{C}(5)-\mathrm{C}(4)-\mathrm{P}(1) & 112.07(14) \\ \mathrm{N}(1)-\mathrm{C}(4)-\mathrm{H}(4) & 105.4 \\ \mathrm{C}(5)-\mathrm{C}(4)-\mathrm{H}(4) & 105.4 \\ \mathrm{P}(1)-\mathrm{C}(4)-\mathrm{H}(4) & 105.4 \\ \mathrm{C}(53)-\mathrm{C}(5)-\mathrm{C}(51) & 107.96(19) \\ \mathrm{C}(53)-\mathrm{C}(5)-\mathrm{C}(52) & 107.46(19) \\ \mathrm{C}(51)-\mathrm{C}(5)-\mathrm{C}(52) & 108.70(19) \\ \mathrm{C}(53)-\mathrm{C}(5)-\mathrm{C}(4) & 108.12(18) \\ \mathrm{C}(51)-\mathrm{C}(5)-\mathrm{C}(4) & 114.04(17) \\ \mathrm{C}(52)-\mathrm{C}(5)-\mathrm{C}(4) & 110.34(17) \\ \mathrm{N}(1)-\mathrm{C}(6)-\mathrm{C}(62) & 107.04(16) \\ \mathrm{N}(1)-\mathrm{C}(6)-\mathrm{C}(63) & 107.73(17) \\ \mathrm{C}(62)-\mathrm{C}(6)-\mathrm{C}(63) & 107.44(19) \\ \mathrm{N}(1)-\mathrm{C}(6)-\mathrm{C}(61) & 114.70(16) \\ \mathrm{C}(62)-\mathrm{C}(6)-\mathrm{C}(61) & 109.89(19) \\ \mathrm{C}(63)-\mathrm{C}(6)-\mathrm{C}(61) & 109.77(17) \\ \mathrm{O}(1)-\mathrm{C}(7)-\mathrm{C}(8) & 107.52(18) \\ \mathrm{O}(1)-\mathrm{C}(7)-\mathrm{H}(7 \mathrm{~A}) & 110.2 \\ \mathrm{C}(8)-\mathrm{C}(7)-\mathrm{H}(7 \mathrm{~A}) & 110.2 \\ \mathrm{O}(1)-\mathrm{C}(7)-\mathrm{H}(7 \mathrm{~B}) & 110.2 \\ \mathrm{C}(8)-\mathrm{C}(7)-\mathrm{H}(7 \mathrm{~B}) & 110.2 \\ \mathrm{H}(7 \mathrm{~A})-\mathrm{C}(7)-\mathrm{H}(7 \mathrm{~B}) & 108.5 \\ \mathrm{C}(5)-\mathrm{C}(51)-\mathrm{H}(51 \mathrm{~A}) & 109.5 \\ \mathrm{C}(5)-\mathrm{C}(51)-\mathrm{H}(51 \mathrm{~B}) & 109.5 \\ \mathrm{H}(51 \mathrm{~A})-\mathrm{C}(51)-\mathrm{H}(51 \mathrm{~B}) & 109.5 \\ \mathrm{C}(5)-\mathrm{C}(51)-\mathrm{H}(51 \mathrm{C}) & 109.5 \\ \mathrm{H}(51 \mathrm{~A})-\mathrm{C}(51)-\mathrm{H}(51 \mathrm{C}) & 109.5 \\ \mathrm{C}(5)-\mathrm{C}(52)-\mathrm{H}(52 \mathrm{~A}) & 109.5 \\ \mathrm{C}(5)-\mathrm{C}(52)-\mathrm{H}(52 \mathrm{~B}) & 109.5 \\ & \end{array}$




$\begin{array}{ll}\mathrm{H}(52 \mathrm{~A})-\mathrm{C}(52)-\mathrm{H}(52 \mathrm{~B}) & 109.5 \\ \mathrm{C}(5)-\mathrm{C}(52)-\mathrm{H}(52 \mathrm{C}) & 109.5 \\ \mathrm{H}(52 \mathrm{~A})-\mathrm{C}(52)-\mathrm{H}(52 \mathrm{C}) & 109.5 \\ \mathrm{H}(52 \mathrm{~B})-\mathrm{C}(52)-\mathrm{H}(52 \mathrm{C}) & 109.5 \\ \mathrm{C}(5)-\mathrm{C}(53)-\mathrm{H}(53 \mathrm{~A}) & 109.5 \\ \mathrm{C}(5)-\mathrm{C}(53)-\mathrm{H}(53 \mathrm{~B}) & 109.5 \\ \mathrm{H}(53 \mathrm{~A})-\mathrm{C}(53)-\mathrm{H}(53 \mathrm{~B}) & 109.5 \\ \mathrm{C}(5)-\mathrm{C}(53)-\mathrm{H}(53 \mathrm{C}) & 109.5 \\ \mathrm{H}(53 \mathrm{~A})-\mathrm{C}(53)-\mathrm{H}(53 \mathrm{C}) & 109.5 \\ \mathrm{H}(53 \mathrm{~B})-\mathrm{C}(53)-\mathrm{H}(53 \mathrm{C}) & 109.5 \\ \mathrm{C}(6)-\mathrm{C}(61)-\mathrm{H}(61 \mathrm{~A}) & 109.5 \\ \mathrm{C}(6)-\mathrm{C}(61)-\mathrm{H}(61 \mathrm{~B}) & 109.5 \\ \mathrm{H}(61 \mathrm{~A})-\mathrm{C}(61)-\mathrm{H}(61 \mathrm{~B}) & 109.5 \\ \mathrm{C}(6)-\mathrm{C}(61)-\mathrm{H}(61 \mathrm{C}) & 109.5 \\ \mathrm{H}(61 \mathrm{~A})-\mathrm{C}(61)-\mathrm{H}(61 \mathrm{C}) & 109.5 \\ \mathrm{H}(61 \mathrm{~B})-\mathrm{C}(61)-\mathrm{H}(61 \mathrm{C}) & 109.5 \\ \mathrm{C}(6)-\mathrm{C}(62)-\mathrm{H}(62 \mathrm{~A}) & 109.5 \\ \mathrm{C}(6)-\mathrm{C}(62)-\mathrm{H}(62 \mathrm{~B}) & 109.5 \\ \mathrm{H}(62 \mathrm{~A})-\mathrm{C}(62)-\mathrm{H}(62 \mathrm{~B}) & 109.5 \\ \mathrm{C}(6)-\mathrm{C}(62)-\mathrm{H}(62 \mathrm{C}) & 109.5 \\ \mathrm{H}(62 \mathrm{~A})-\mathrm{C}(62)-\mathrm{H}(62 \mathrm{C}) & 109.5 \\ \mathrm{H}(62 \mathrm{~B})-\mathrm{C}(62)-\mathrm{H}(62 \mathrm{C}) & 109.5 \\ \mathrm{C}(6)-\mathrm{C}(63)-\mathrm{H}(63 \mathrm{~A}) & 109.5 \\ \mathrm{C}(6)-\mathrm{C}(63)-\mathrm{H}(63 \mathrm{~B}) & 109.5 \\ \mathrm{H}(63 \mathrm{~A})-\mathrm{C}(63)-\mathrm{H}(63 \mathrm{~B}) & 109.5 \\ \mathrm{C}(6)-\mathrm{C}(63)-\mathrm{H}(63 \mathrm{C}) & 109.5 \\ \mathrm{H}(63 \mathrm{~A})-\mathrm{C}(63)-\mathrm{H}(63 \mathrm{C}) & 109.5 \\ \mathrm{H}(63 \mathrm{~B})-\mathrm{C}(63)-\mathrm{H}(63 \mathrm{C}) & 109.5 \\ \mathrm{O}(3)-\mathrm{N}(1)-\mathrm{C}(4) & 106.74(13) \\ \mathrm{O}(3)-\mathrm{N}(1)-\mathrm{C}(6) & 108.07(14) \\ \mathrm{C}(4)-\mathrm{N}(1)-\mathrm{C}(6) & 115.99(15) \\ \mathrm{C}(1)-\mathrm{O}(1)-\mathrm{C}(7) & 115.95(15) \\ \mathrm{C}(2)-\mathrm{O}(3)-\mathrm{N}(1) & 111.11(13) \\ \mathrm{C}(11)-\mathrm{O}(4)-\mathrm{P}(1) & 123.54(15) \\ \mathrm{C}(9)-\mathrm{O}(6)-\mathrm{P}(1) & 118.93(13) \\ \mathrm{O}(5)-\mathrm{P}(1)-\mathrm{O}(4) & 113.60(9) \\ \mathrm{O}(5)-\mathrm{P}(1)-\mathrm{O}(6) & 115.38(8) \\ \mathrm{O}(4)-\mathrm{P}(1)-\mathrm{O}(6) & 100.92(8) \\ \mathrm{O}(5)-\mathrm{P}(1)-\mathrm{C}(4) & 116.88(9) \\ \mathrm{O}(6)-\mathrm{P}(1)-\mathrm{C}(4) & 110.46(8) \\ & 97.47(8) \\ & \\ & \end{array}$

Symmetry transformations used to generate equivalent atoms:

$\# 1-x+1 / 2,-y+3 / 2,-z$ 
Table 4. Anisotropic displacement parameters $\left(\AA^{2} \mathrm{x} 10^{3}\right)$ for $\mathrm{DEPN}_{2}$. The anisotropic displacement factor exponent takes the form: $-2 \pi^{2}\left[h^{2} a^{* 2} U^{11}+\ldots+2 h k\right.$ $\left.a^{*} b^{*} U^{12}\right]$

\begin{tabular}{|c|c|c|c|c|c|c|}
\hline & $\mathrm{U}^{11}$ & $\mathrm{U}^{22}$ & $\mathrm{U}^{33}$ & $\mathrm{U}^{23}$ & $\mathrm{U}^{13}$ & $\mathrm{U}^{12}$ \\
\hline $\mathrm{C}(8)$ & $56(2)$ & $43(1)$ & $44(1)$ & $-1(1)$ & $32(1)$ & $-11(1)$ \\
\hline $\mathrm{C}(9)$ & $44(1)$ & $41(1)$ & $37(1)$ & $4(1)$ & $26(1)$ & $3(1)$ \\
\hline $\mathrm{C}(10)$ & $64(2)$ & $45(2)$ & $59(2)$ & $14(1)$ & $43(2)$ & $0(1)$ \\
\hline $\mathrm{C}(11)$ & $91(2)$ & $40(1)$ & $32(1)$ & $-3(1)$ & $2(1)$ & $-31(2)$ \\
\hline $\mathrm{C}(12)$ & $53(2)$ & \multicolumn{2}{|c|}{$117(3) 60(2)$} & $-45(2)$ & $27(2)$ & $-32(2)$ \\
\hline $\mathrm{C}(1)$ & $28(1)$ & $16(1)$ & $31(1)$ & $3(1)$ & $12(1)$ & $2(1)$ \\
\hline $\mathrm{C}(2)$ & $25(1)$ & $18(1)$ & $25(1)$ & $5(1)$ & $9(1)$ & $0(1)$ \\
\hline$C(3)$ & $29(1)$ & $18(1)$ & $30(1)$ & $1(1)$ & $15(1)$ & $-1(1)$ \\
\hline$C(4)$ & $26(1)$ & $17(1)$ & $29(1)$ & $0(1)$ & $12(1)$ & $0(1)$ \\
\hline$C(5)$ & $27(1)$ & $28(1)$ & $38(1)$ & $2(1)$ & $7(1)$ & $-2(1)$ \\
\hline$C(6)$ & $40(1)$ & $20(1)$ & $45(1)$ & $4(1)$ & $28(1)$ & $6(1)$ \\
\hline$C(7)$ & $30(1)$ & $36(1)$ & $45(1)$ & $4(1)$ & $23(1)$ & $-5(1)$ \\
\hline $\mathrm{C}(51)$ & $25(1)$ & $35(1)$ & $48(1)$ & $10(1)$ & $5(1)$ & $0(1)$ \\
\hline$C(52)$ & $25(1)$ & $33(1)$ & $68(2)$ & $11(1)$ & $12(1)$ & $-4(1)$ \\
\hline $\mathrm{C}(53)$ & $49(2)$ & $49(2)$ & $34(1)$ & $-6(1)$ & $-1(1)$ & $-4(1)$ \\
\hline $\mathrm{C}(61)$ & $30(1)$ & $24(1)$ & $49(1)$ & $4(1)$ & $18(1)$ & $7(1)$ \\
\hline$C(62)$ & $53(2)$ & $33(1)$ & $72(2)$ & $13(1)$ & $44(1)$ & $14(1)$ \\
\hline $\mathrm{C}(63)$ & $65(2)$ & $26(1)$ & $44(1)$ & $0(1)$ & $33(1)$ & $7(1)$ \\
\hline $\mathrm{N}(1)$ & $28(1)$ & $16(1)$ & $31(1)$ & $-1(1)$ & $14(1)$ & $-1(1)$ \\
\hline $\mathrm{O}(1)$ & $27(1)$ & $24(1)$ & $31(1)$ & $3(1)$ & $15(1)$ & $-2(1)$ \\
\hline $\mathrm{O}(2)$ & $26(1)$ & $37(1)$ & $35(1)$ & $7(1)$ & $7(1)$ & $-4(1)$ \\
\hline $\mathrm{O}(3)$ & $27(1)$ & $15(1)$ & $28(1)$ & $2(1)$ & $10(1)$ & $-4(1)$ \\
\hline $\mathrm{O}(4)$ & $38(1)$ & $25(1)$ & $27(1)$ & $-2(1)$ & $11(1)$ & $-5(1)$ \\
\hline $\mathrm{O}(5)$ & $53(1)$ & $23(1)$ & $50(1)$ & $2(1)$ & $31(1)$ & $7(1)$ \\
\hline $\mathrm{O}(6)$ & $42(1)$ & $23(1)$ & $38(1)$ & $2(1)$ & $25(1)$ & $-1(1)$ \\
\hline $\mathrm{P}(1)$ & $33(1)$ & $18(1)$ & $32(1)$ & 1(1) & $18(1)$ & 1(1) \\
\hline
\end{tabular}


Table 5. Hydrogen coordinates ( $\mathrm{x} 10^{4}$ ) and isotropic displacement parameters $\left(\AA^{2} \times 10^{3}\right)$ for $\mathrm{DEPN}_{2}$.

\begin{tabular}{lllll}
\hline & x & y & z & U(eq) \\
\hline $\mathrm{H}(8 \mathrm{~A})$ & 3607 & 6932 & -2319 & 66 \\
$\mathrm{H}(8 \mathrm{~B})$ & 4223 & 7754 & -2068 & 66 \\
$\mathrm{H}(8 \mathrm{C})$ & 3351 & 7909 & -2152 & 66 \\
$\mathrm{H}(9 \mathrm{~A})$ & 1258 & 4441 & 2018 & 45 \\
$\mathrm{H}(9 \mathrm{~B})$ & 2132 & 4051 & 2647 & 45 \\
$\mathrm{H}(10 \mathrm{~A})$ & 812 & 2945 & 1577 & 76 \\
$\mathrm{H}(10 \mathrm{~B})$ & 1102 & 3086 & 2577 & 76 \\
$\mathrm{H}(10 \mathrm{C})$ & 1672 & 2586 & 2269 & 76 \\
$\mathrm{H}(11 \mathrm{~A})$ & 3240 & 5908 & 2522 & 78 \\
$\mathrm{H}(11 \mathrm{~B})$ & 3627 & 5921 & 1885 & 78 \\
$\mathrm{H}(12 \mathrm{~A})$ & 4209 & 4881 & 3406 & 115 \\
$\mathrm{H}(12 \mathrm{~B})$ & 4638 & 5759 & 3289 & 115 \\
$\mathrm{H}(12 \mathrm{C})$ & 4586 & 4863 & 2763 & 115 \\
$\mathrm{H}(2)$ & 2448 & 6199 & -717 & 29 \\
$\mathrm{H}(3 \mathrm{~A})$ & 3058 & 7217 & 849 & 31 \\
$\mathrm{H}(3 \mathrm{~B})$ & 2153 & 6868 & 344 & 31 \\
$\mathrm{H}(4)$ & 2101 & 3748 & 161 & 29 \\
$\mathrm{H}(7 \mathrm{~A})$ & 4576 & 6847 & -860 & 42 \\
$\mathrm{H}(7 \mathrm{~B})$ & 4291 & 7815 & -700 & 42 \\
$\mathrm{H}(51 \mathrm{~A})$ & 1288 & 5845 & -1046 & 62 \\
$\mathrm{H}(51 \mathrm{~B})$ & 1017 & 5892 & -315 & 62 \\
$\mathrm{H}(51 \mathrm{C})$ & 381 & 5627 & -1273 & 62 \\
$\mathrm{H}(52 \mathrm{~A})$ & 12 & 4177 & -896 & 69 \\
$\mathrm{H}(52 \mathrm{~B})$ & 546 & 4461 & 84 & 69 \\
$\mathrm{H}(52 \mathrm{C})$ & 690 & 3511 & -247 & 69 \\
$\mathrm{H}(53 \mathrm{~A})$ & 535 & 4204 & -1904 & 78 \\
$\mathrm{H}(53 \mathrm{~B})$ & 1191 & 3476 & -1329 & 78 \\
$\mathrm{H}(53 \mathrm{C})$ & 1464 & 4387 & -1600 & 78 \\
$\mathrm{H}(61 \mathrm{~A})$ & 4010 & 4177 & 1309 & 52 \\
$\mathrm{H}(61 \mathrm{~B})$ & 4152 & 3263 & 921 & 52 \\
$\mathrm{H}(61 \mathrm{C})$ & 3369 & 3379 & 1042 & 52 \\
$\mathrm{H}(62 \mathrm{~A})$ & 3577 & 4907 & -770 & 70 \\
$\mathrm{H}(62 \mathrm{~B})$ & 4230 & 4136 & -305 & 70 \\
$\mathrm{H}(62 \mathrm{C})$ & 4201 & 4996 & 225 & 70 \\
$\mathrm{H}(63 \mathrm{~A})$ & 2511 & 3016 & -546 & 63 \\
$\mathrm{H}(63 \mathrm{~B})$ & 3328 & 2893 & -600 & 63 \\
$\mathrm{H}(63 \mathrm{C})$ & 2656 & 3574 & -1235 & 63 \\
& & & & \\
\hline
\end{tabular}


Table 6. Torsion angles $\left[{ }^{\circ}\right]$ for $\mathrm{DEPN}_{2}$.

\begin{tabular}{ll}
\hline $\mathrm{O}(2)-\mathrm{C}(1)-\mathrm{C}(2)-\mathrm{O}(3)$ & $-49.1(3)$ \\
$\mathrm{O}(1)-\mathrm{C}(1)-\mathrm{C}(2)-\mathrm{O}(3)$ & $134.91(16)$ \\
$\mathrm{O}(2)-\mathrm{C}(1)-\mathrm{C}(2)-\mathrm{C}(3)$ & $69.5(2)$ \\
$\mathrm{O}(1)-\mathrm{C}(1)-\mathrm{C}(2)-\mathrm{C}(3)$ & $-106.51(17)$ \\
$\mathrm{O}(3)-\mathrm{C}(2)-\mathrm{C}(3)-\mathrm{C}(3) \# 1$ & $-175.16(19)$ \\
$\mathrm{C}(1)-\mathrm{C}(2)-\mathrm{C}(3)-\mathrm{C}(3) \# 1$ & $63.8(3)$ \\
$\mathrm{N}(1)-\mathrm{C}(4)-\mathrm{C}(5)-\mathrm{C}(53)$ & $56.3(2)$ \\
$\mathrm{P}(1)-\mathrm{C}(4)-\mathrm{C}(5)-\mathrm{C}(53)$ & $-171.61(15)$ \\
$\mathrm{N}(1)-\mathrm{C}(4)-\mathrm{C}(5)-\mathrm{C}(51)$ & $-63.8(2)$ \\
$\mathrm{P}(1)-\mathrm{C}(4)-\mathrm{C}(5)-\mathrm{C}(51)$ & $68.3(2)$ \\
$\mathrm{N}(1)-\mathrm{C}(4)-\mathrm{C}(5)-\mathrm{C}(52)$ & $173.55(17)$ \\
$\mathrm{P}(1)-\mathrm{C}(4)-\mathrm{C}(5)-\mathrm{C}(52)$ & $-54.4(2)$ \\
$\mathrm{C}(5)-\mathrm{C}(4)-\mathrm{N}(1)-\mathrm{O}(3)$ & $110.19(16)$ \\
$\mathrm{P}(1)-\mathrm{C}(4)-\mathrm{N}(1)-\mathrm{O}(3)$ & $-19.84(18)$ \\
$\mathrm{C}(5)-\mathrm{C}(4)-\mathrm{N}(1)-\mathrm{C}(6)$ & $-129.35(18)$ \\
$\mathrm{P}(1)-\mathrm{C}(4)-\mathrm{N}(1)-\mathrm{C}(6)$ & $100.63(17)$ \\
$\mathrm{C}(62)-\mathrm{C}(6)-\mathrm{N}(1)-\mathrm{O}(3)$ & $-56.5(2)$ \\
$\mathrm{C}(63)-\mathrm{C}(6)-\mathrm{N}(1)-\mathrm{O}(3)$ & $-171.80(15)$ \\
$\mathrm{C}(61)-\mathrm{C}(6)-\mathrm{N}(1)-\mathrm{O}(3)$ & $65.7(2)$ \\
$\mathrm{C}(62)-\mathrm{C}(6)-\mathrm{N}(1)-\mathrm{C}(4)$ & $-176.27(18)$ \\
$\mathrm{C}(63)-\mathrm{C}(6)-\mathrm{N}(1)-\mathrm{C}(4)$ & $68.5(2)$ \\
$\mathrm{C}(61)-\mathrm{C}(6)-\mathrm{N}(1)-\mathrm{C}(4)$ & $-54.1(2)$ \\
$\mathrm{O}(2)-\mathrm{C}(1)-\mathrm{O}(1)-\mathrm{C}(7)$ & $-4.6(3)$ \\
$\mathrm{C}(2)-\mathrm{C}(1)-\mathrm{O}(1)-\mathrm{C}(7)$ & $171.42(16)$ \\
$\mathrm{C}(8)-\mathrm{C}(7)-\mathrm{O}(1)-\mathrm{C}(1)$ & $-176.37(17)$ \\
$\mathrm{C}(1)-\mathrm{C}(2)-\mathrm{O}(3)-\mathrm{N}(1)$ & $-108.26(17)$ \\
$\mathrm{C}(3)-\mathrm{C}(2)-\mathrm{O}(3)-\mathrm{N}(1)$ & $133.27(15)$ \\
$\mathrm{C}(4)-\mathrm{N}(1)-\mathrm{O}(3)-\mathrm{C}(2)$ & $-117.42(15)$ \\
$\mathrm{C}(6)-\mathrm{N}(1)-\mathrm{O}(3)-\mathrm{C}(2)$ & $117.17(16)$ \\
$\mathrm{C}(12)-\mathrm{C}(11)-\mathrm{O}(4)-\mathrm{P}(1)$ & $170.14(18)$ \\
$\mathrm{C}(10)-\mathrm{C}(9)-\mathrm{O}(6)-\mathrm{P}(1)$ & $175.39(15)$ \\
$\mathrm{C}(11)-\mathrm{O}(4)-\mathrm{P}(1)-\mathrm{O}(5)$ & $-17.3(2)$ \\
$\mathrm{C}(11)-\mathrm{O}(4)-\mathrm{P}(1)-\mathrm{O}(6)$ & $-141.38(18)$ \\
$\mathrm{C}(11)-\mathrm{O}(4)-\mathrm{P}(1)-\mathrm{C}(4)$ & $116.30(19)$ \\
$\mathrm{C}(9)-\mathrm{O}(6)-\mathrm{P}(1)-\mathrm{O}(5)$ & $-42.64(18)$ \\
$\mathrm{C}(9)-\mathrm{O}(6)-\mathrm{P}(1)-\mathrm{O}(4)$ & $80.23(16)$ \\
$\mathrm{C}(9)-\mathrm{O}(6)-\mathrm{P}(1)-\mathrm{C}(4)$ & $-167.17(15)$ \\
$\mathrm{N}(1)-\mathrm{C}(4)-\mathrm{P}(1)-\mathrm{O}(5)$ & $90.12(15)$ \\
$\mathrm{C}(5)-\mathrm{C}(4)-\mathrm{P}(1)-\mathrm{O}(5)$ & $-39.64(16)$ \\
$\mathrm{C}(1)-\mathrm{C}(4)-\mathrm{P}(1)-\mathrm{O}(4)$ & $-41.79(16)$ \\
& $-171.56(12)$ \\
& \\
&
\end{tabular}


$\mathrm{N}(1)-\mathrm{C}(4)-\mathrm{P}(1)-\mathrm{O}(6) \quad-146.44(14)$

$\mathrm{C}(5)-\mathrm{C}(4)-\mathrm{P}(1)-\mathrm{O}(6) \quad 83.80(14)$

Symmetry transformations used to generate equivalent atoms:

$\# 1-x+1 / 2,-y+3 / 2,-z$ 


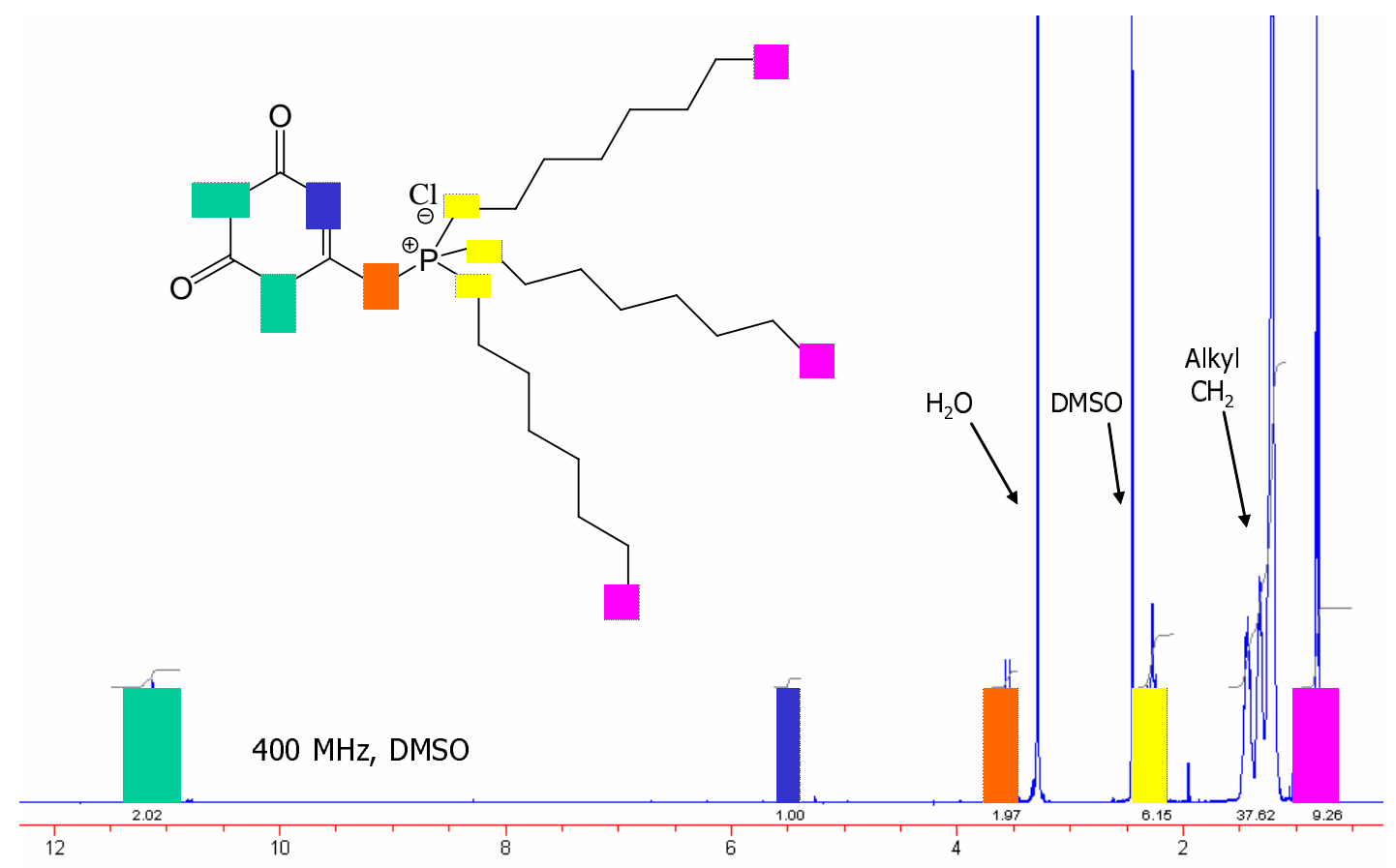

Figure 1. ${ }^{1} \mathrm{H}$ NMR Spectrum of $\mathrm{UP}^{+}\left(400 \mathrm{MHz}, \mathrm{DMSO}-\mathrm{d}_{6}, 25^{\circ} \mathrm{C}\right)$.

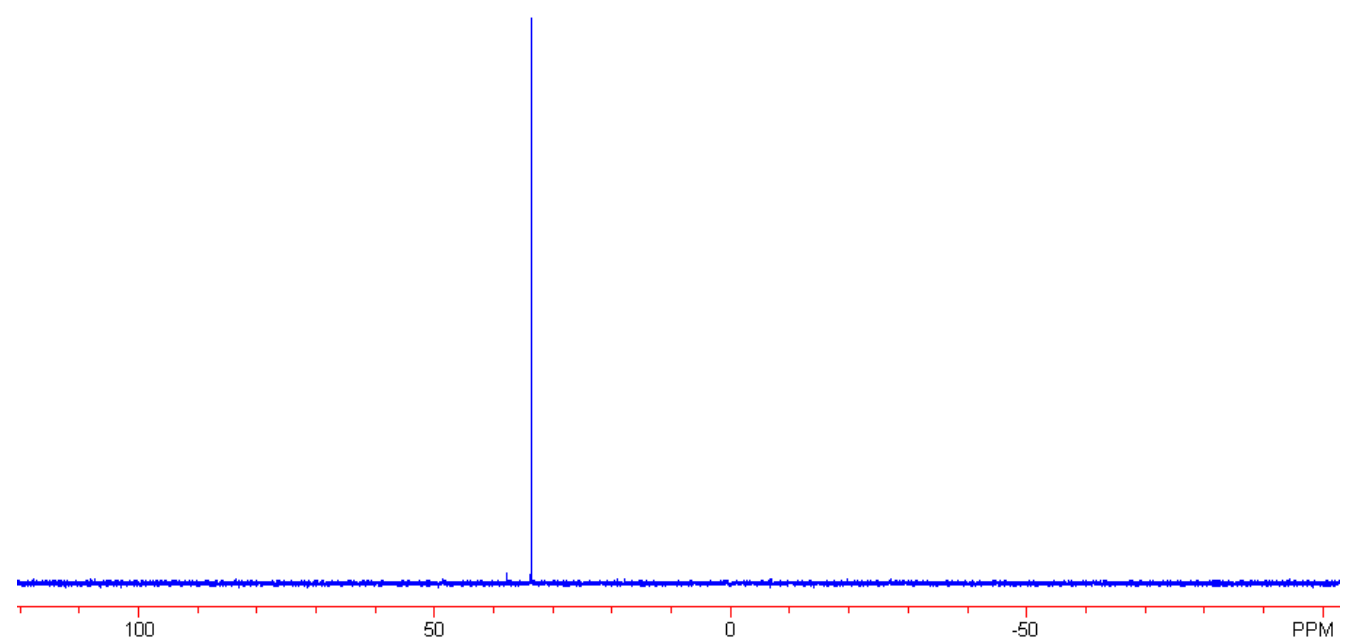

Figure 2. ${ }^{31} \mathrm{P} \mathrm{NMR}$ of $\mathrm{UP}^{+}\left(\mathrm{DMSO}-\mathrm{d}_{6}, 162 \mathrm{MHz}, 25^{\circ} \mathrm{C}\right)$. 


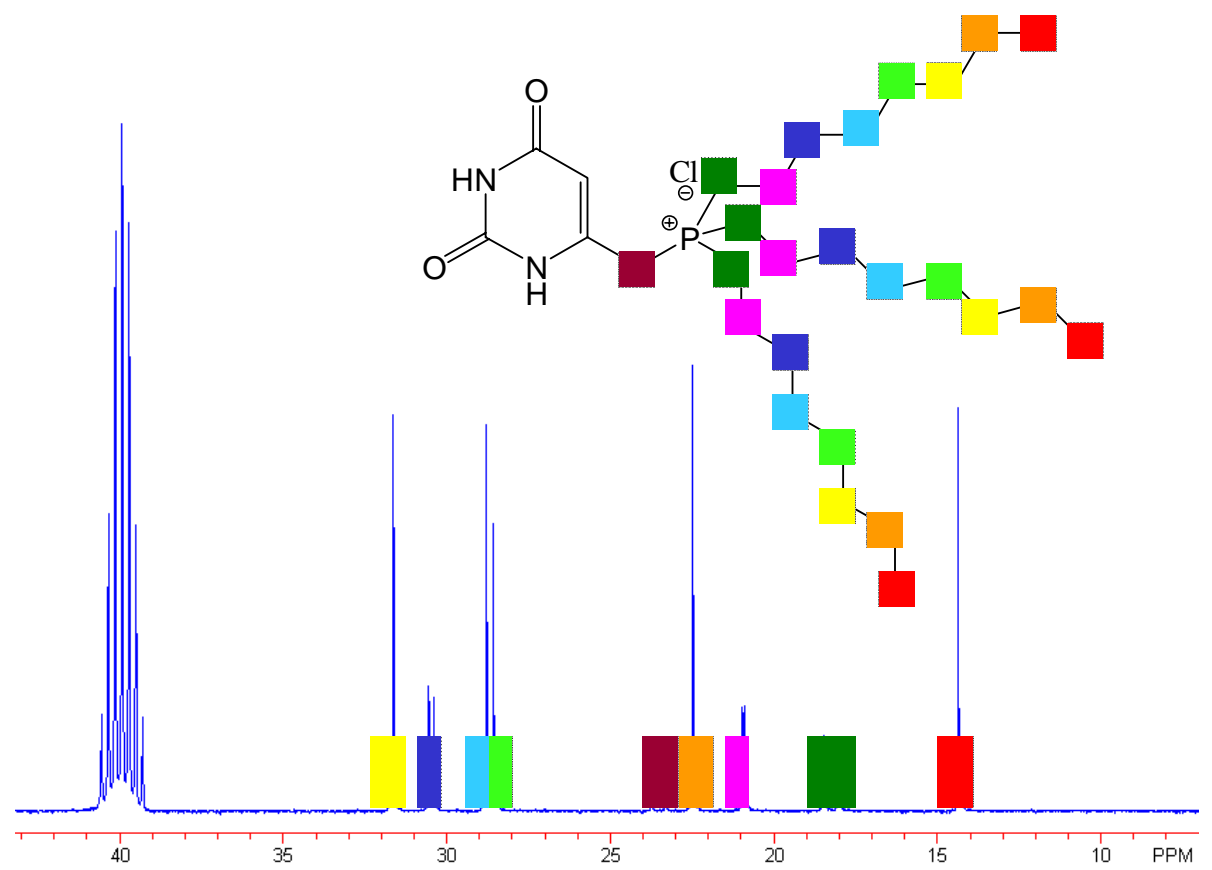

Figure 3. ${ }^{31} \mathrm{C} \mathrm{NMR}$ of $\mathrm{UP}^{+}\left(\mathrm{DMSO}_{-} \mathrm{d}_{6}, 101 \mathrm{MHz}, 25{ }^{\circ} \mathrm{C}\right)$.
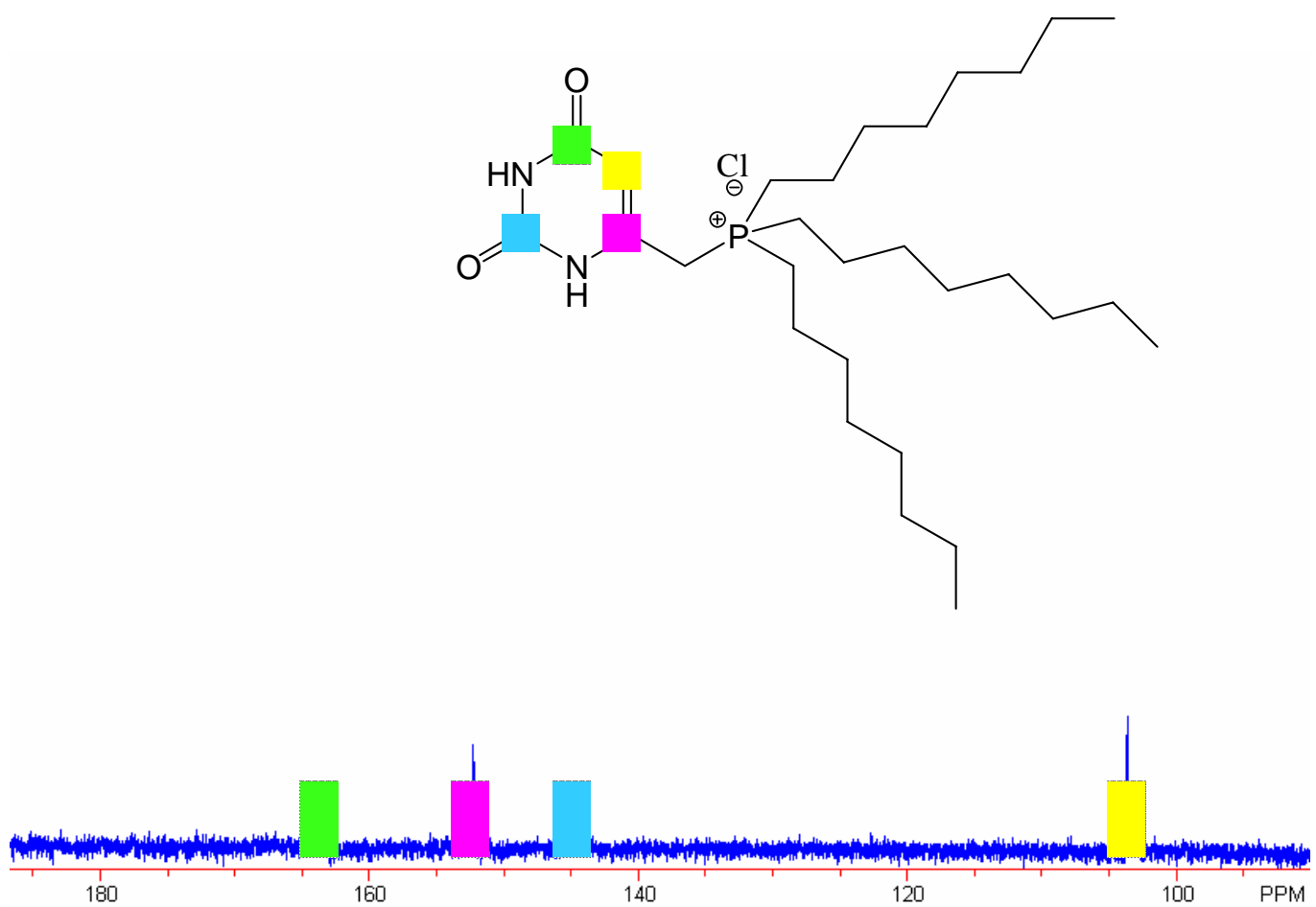

Figure $4 .{ }^{13} \mathrm{C} \mathrm{NMR}$ of $\mathrm{UP}^{+}$continued. 


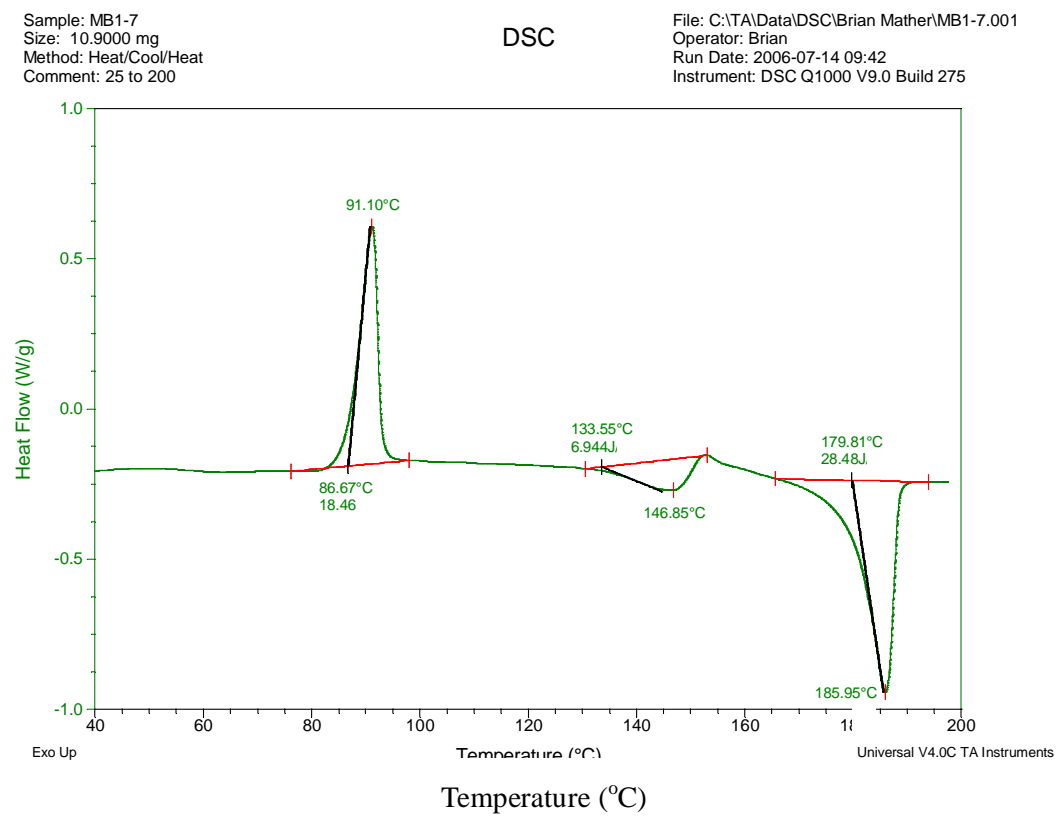

Figure 5. DSC of $\mathrm{UP}^{+} .2^{\text {nd }}$ heat $10{ }^{\circ} \mathrm{C} / \mathrm{min}, \mathrm{N}_{2}$ 


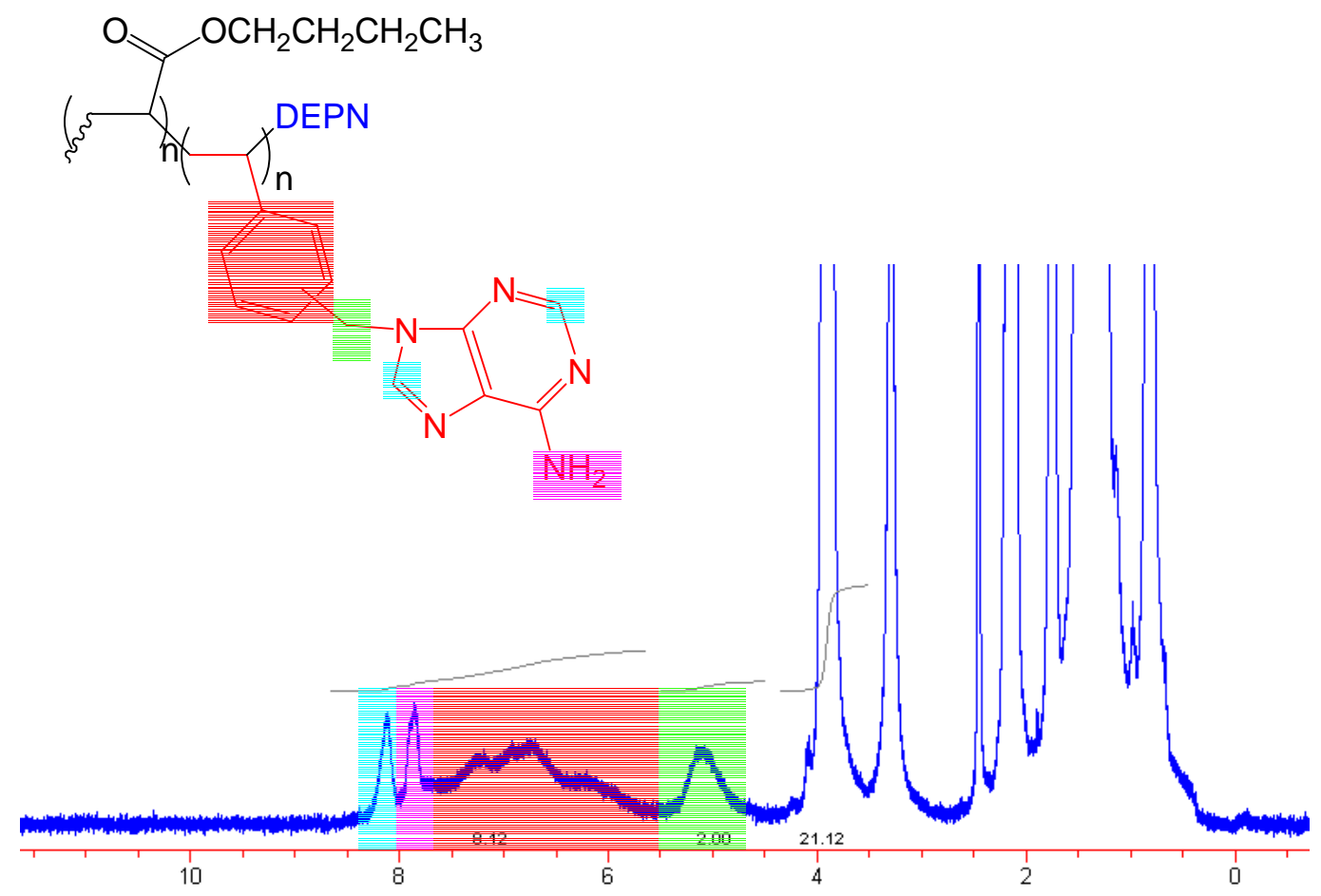

Figure 6. ${ }^{1} \mathrm{H}$ NMR of Adenine Functional Triblock Copolymer (400 MHz, DMSO-d : $\mathrm{CDCl}_{3} 1: 1,25^{\circ} \mathrm{C}$ )

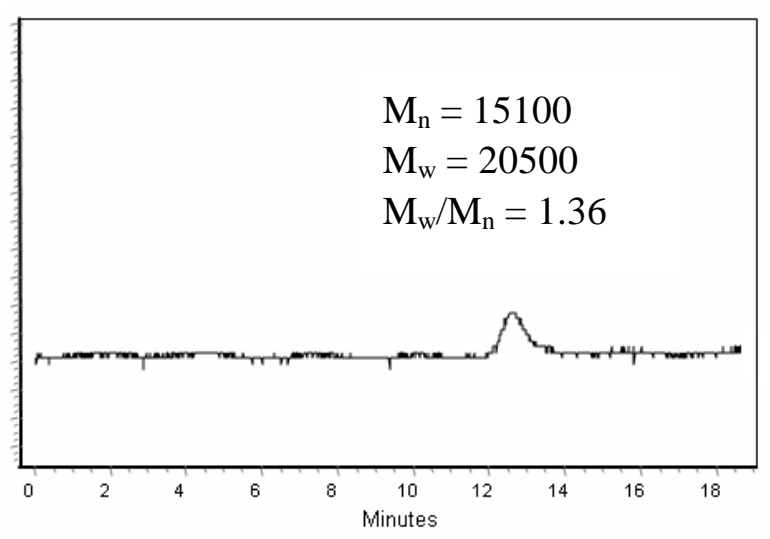

Figure 7. SEC of Adenine functional triblock copolymer. SEC: THF, $30{ }^{\circ} \mathrm{C}$, DRI, polystyrene equivalent molecular weights. 


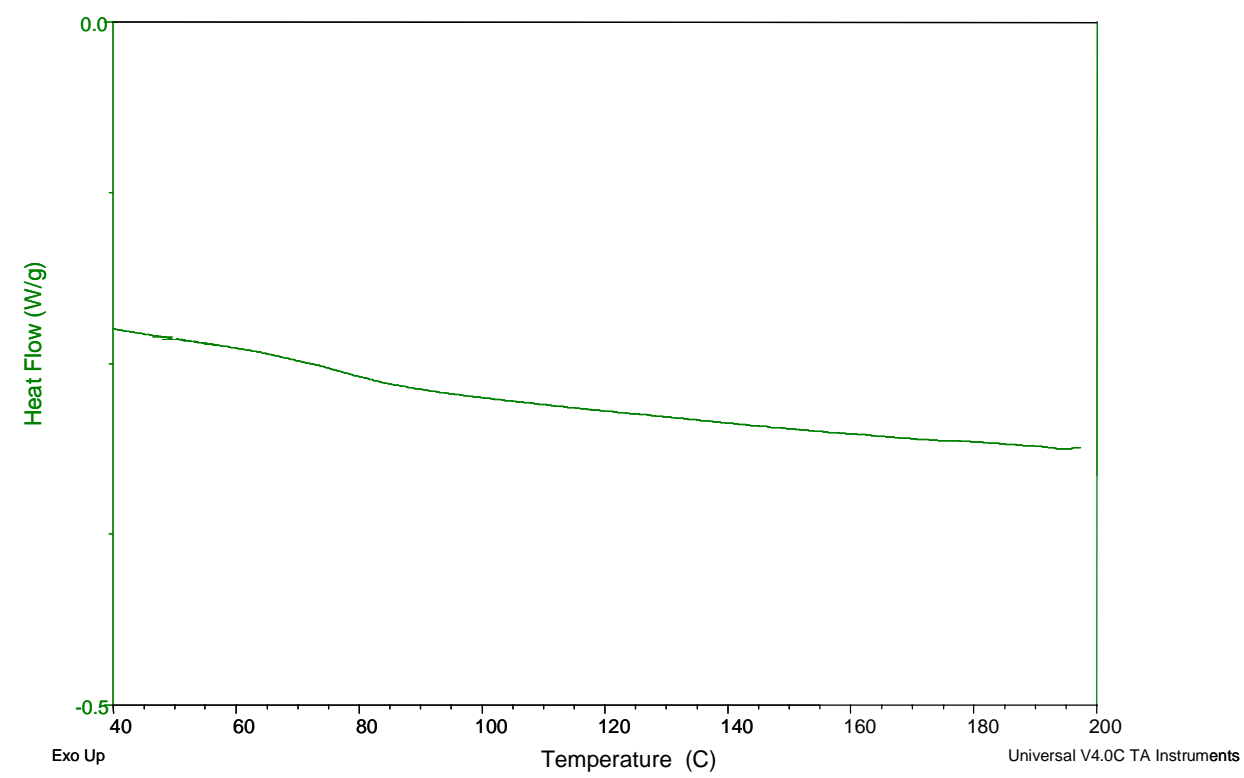

Figure 8. DSC of $\mathrm{UP}^{+} /$Adenine functional block copolymer blend, 1:1 A:U molar ratio. $2^{\text {nd }}$ heat $10{ }^{\circ} \mathrm{C} / \mathrm{min}, \mathrm{N}_{2}$.

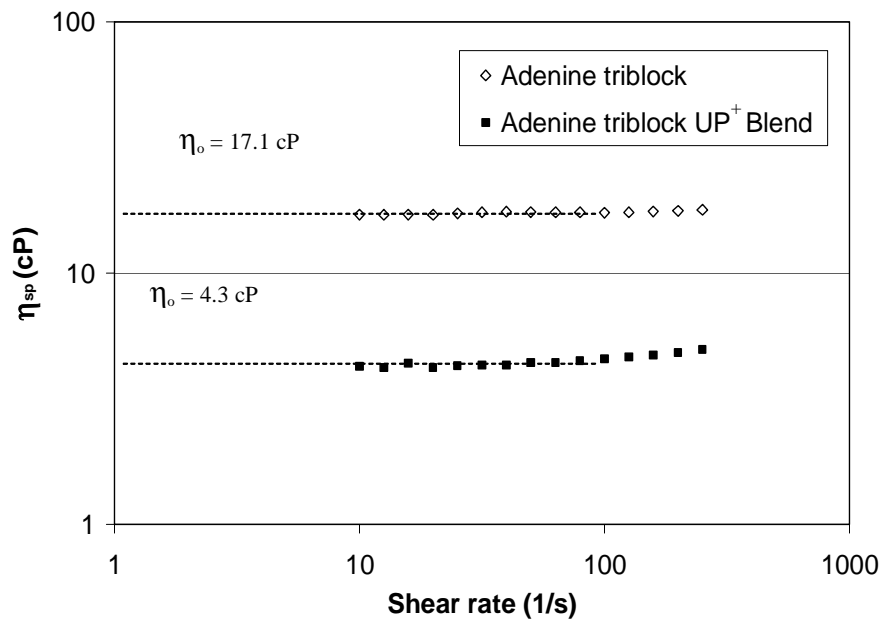

Figure 9. Solution viscosity measurements for Adenine polymer and $\mathrm{UP}^{+}$Blend in chloroform. Conditions: chloroform, $4.2 \mathrm{wt} \%, 25^{\circ} \mathrm{C}$. 


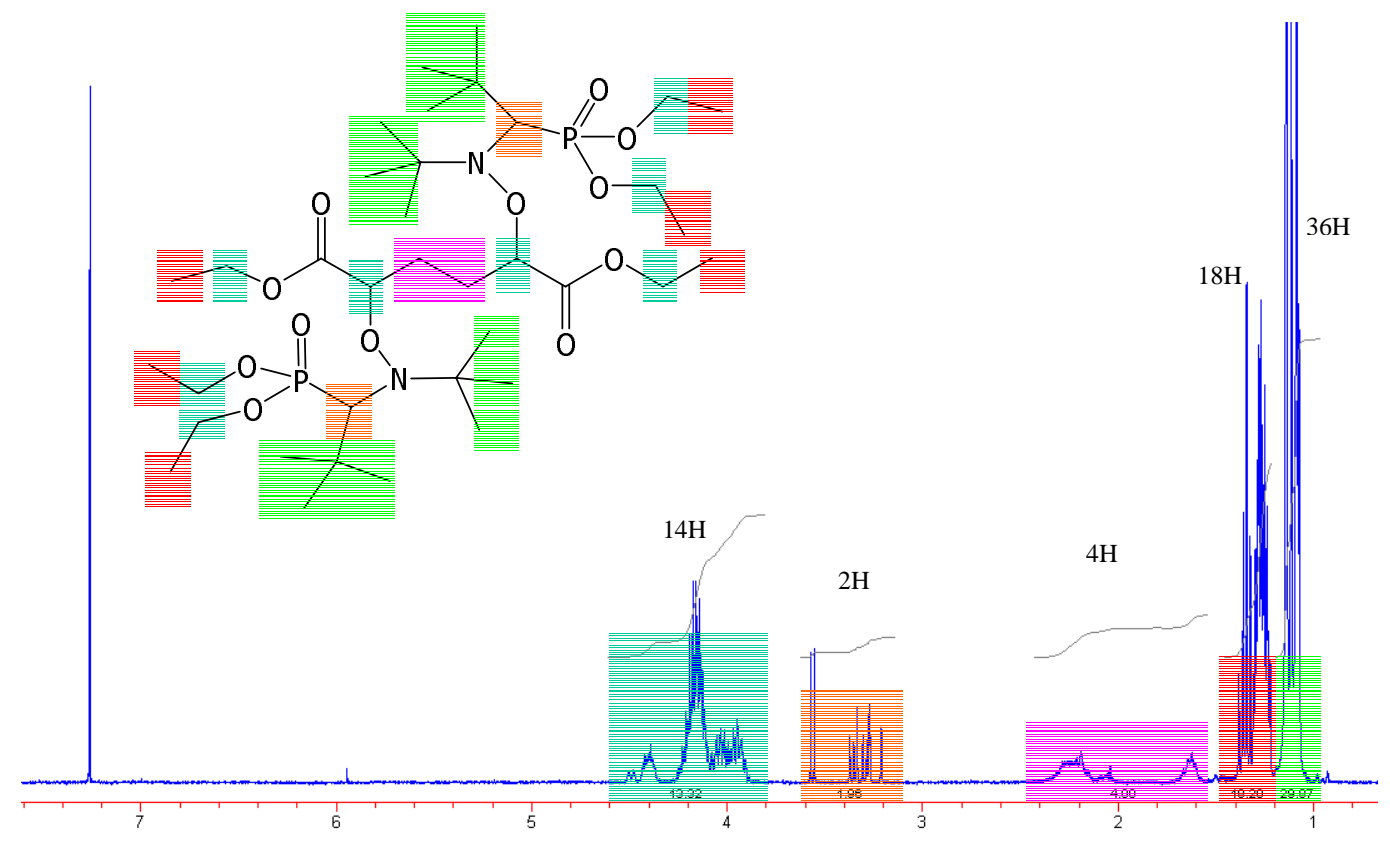

Figure 10. ${ }^{1} \mathrm{H}$ NMR Spectrum of $\mathrm{DEPN}_{2}\left(400 \mathrm{MHz}, \mathrm{CDCl}_{3}, 25{ }^{\circ} \mathrm{C}\right)$.

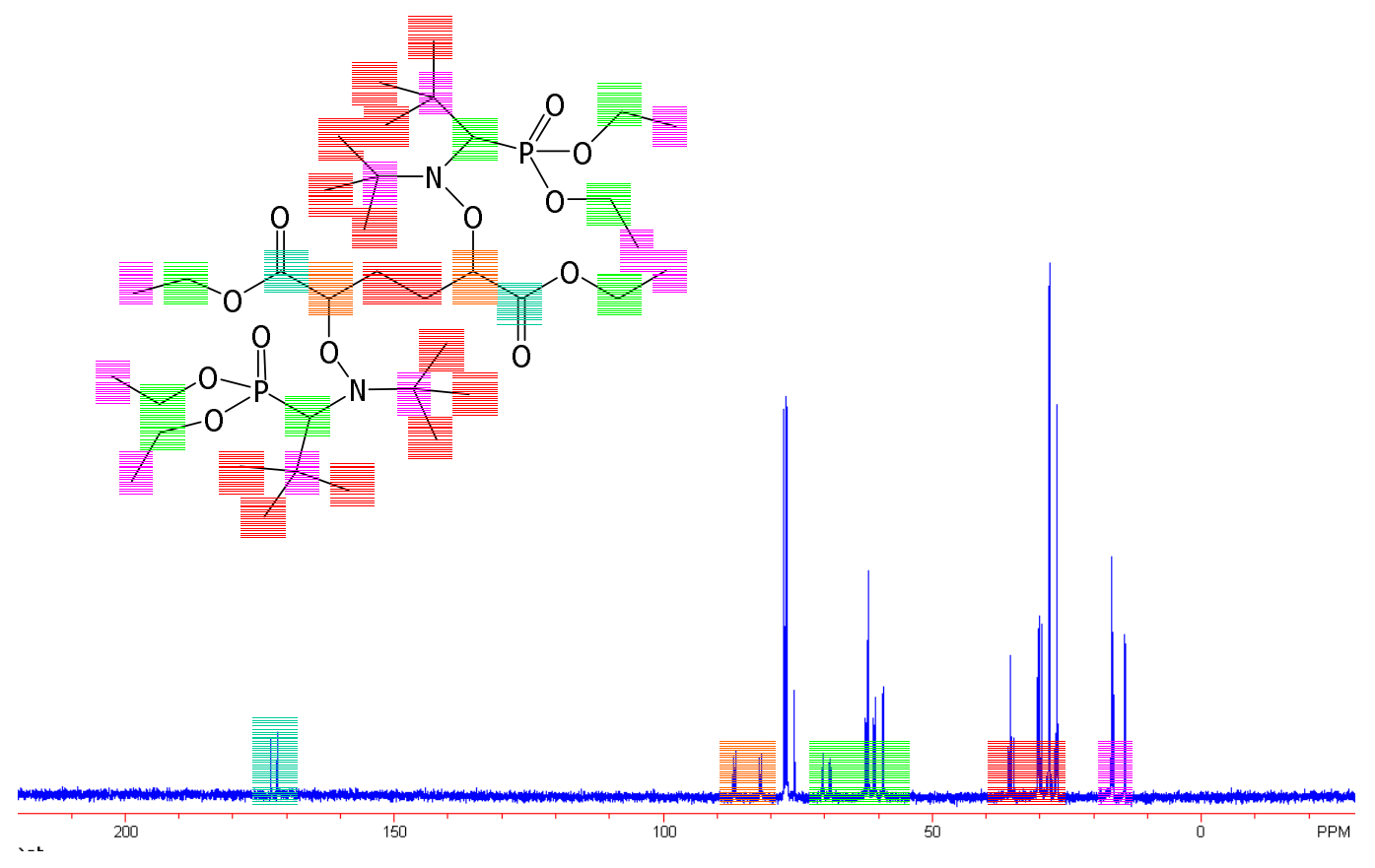

Figure 11. ${ }^{13} \mathrm{C}$ NMR Spectrum of $\mathrm{DEPN}_{2}\left(101 \mathrm{MHz}, \mathrm{CDCl}_{3}, 25{ }^{\circ} \mathrm{C}\right)$ 


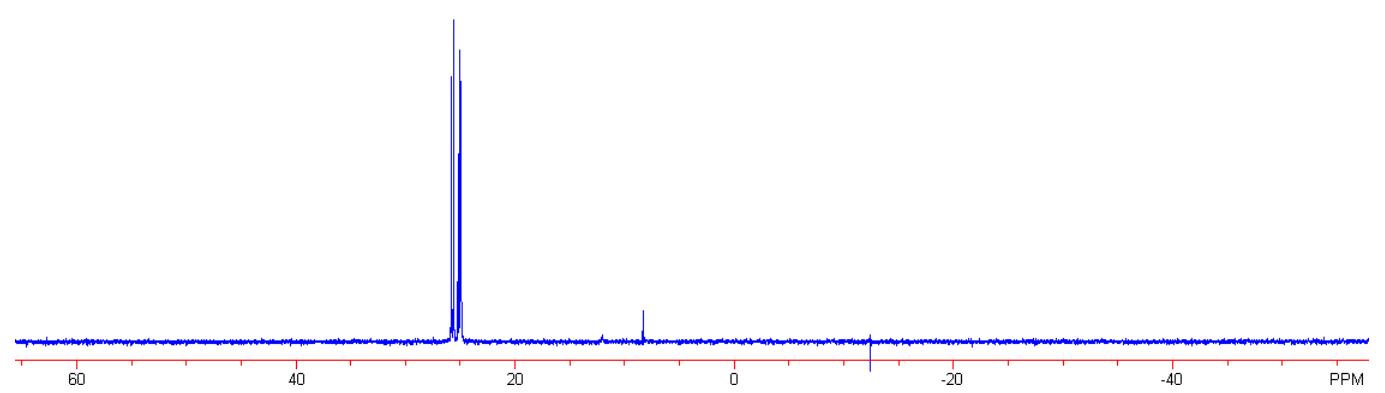

Figure 12. ${ }^{31} \mathrm{P} \mathrm{NMR}$ of $\mathrm{DEPN}_{2}\left(162 \mathrm{MHz}, \mathrm{CDCl}_{3}, 25^{\circ} \mathrm{C}\right)$.

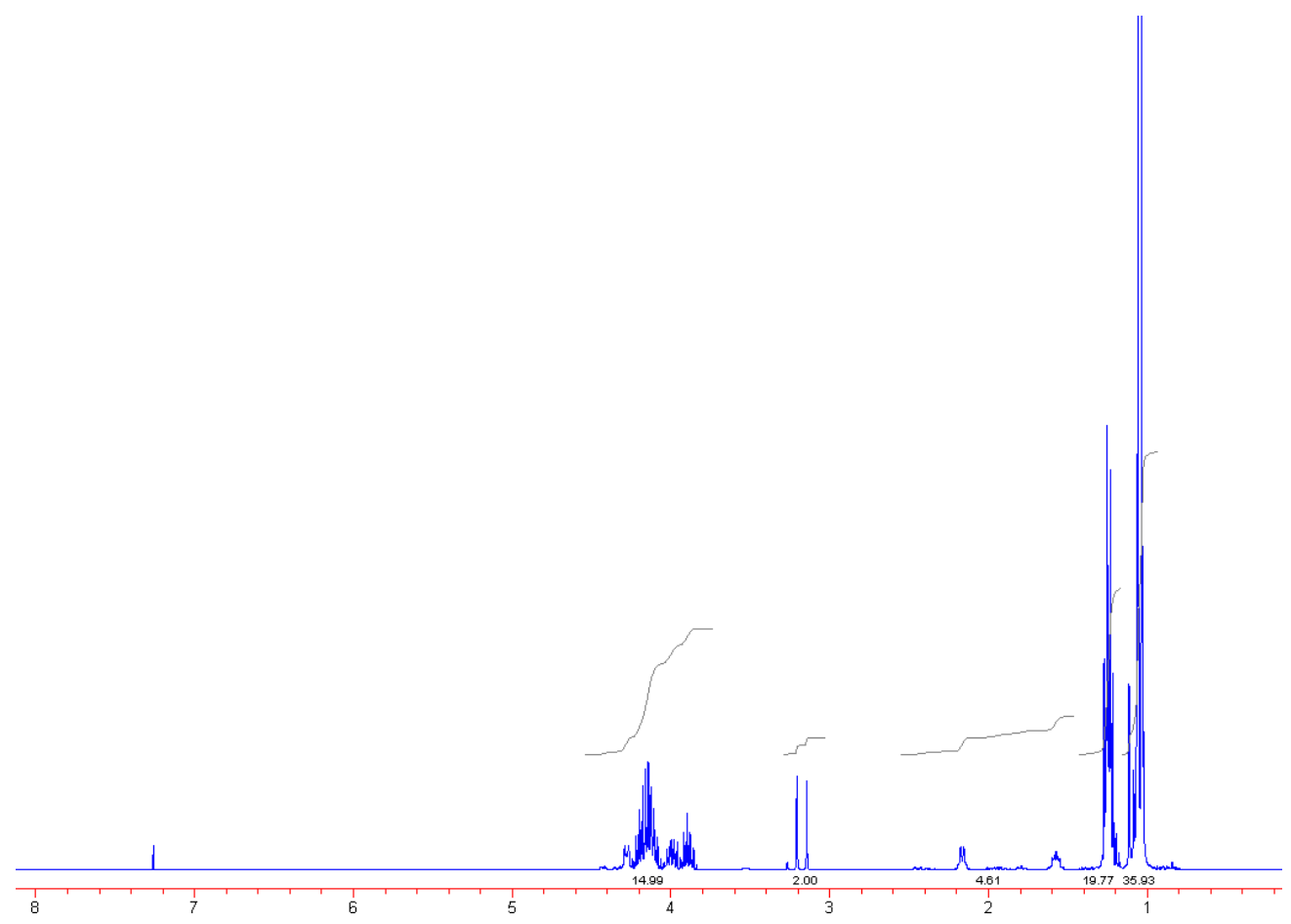

Figure 13. ${ }^{1} \mathrm{H}$ NMR of meso diastereomer of $\mathrm{DEPN}_{2}\left(400 \mathrm{MHz}, \mathrm{CDCl}_{3}, 25{ }^{\circ} \mathrm{C}\right)$. 


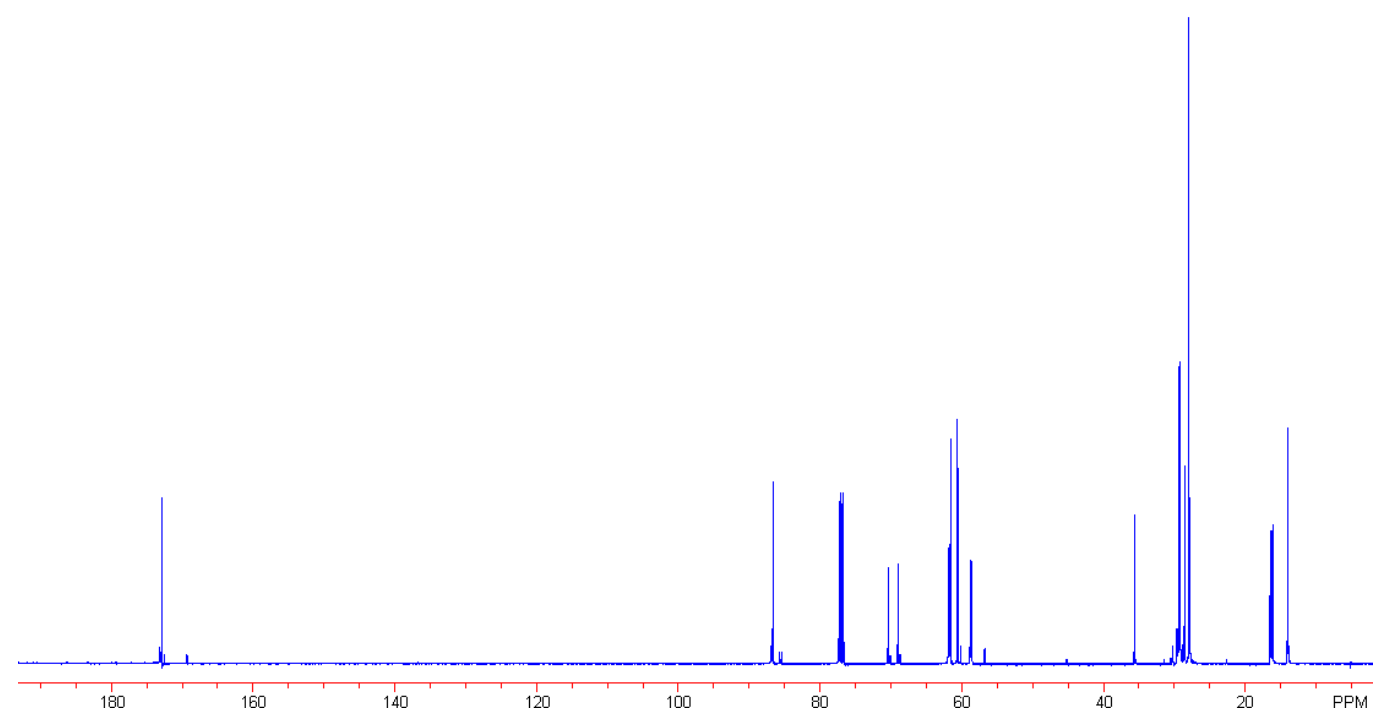

Figure 14. ${ }^{13} \mathrm{C} \mathrm{NMR}$ of meso diastereomer of $\mathrm{DEPN}_{2}\left(400 \mathrm{MHz}, \mathrm{CDCl}_{3}, 25^{\circ} \mathrm{C}\right)$

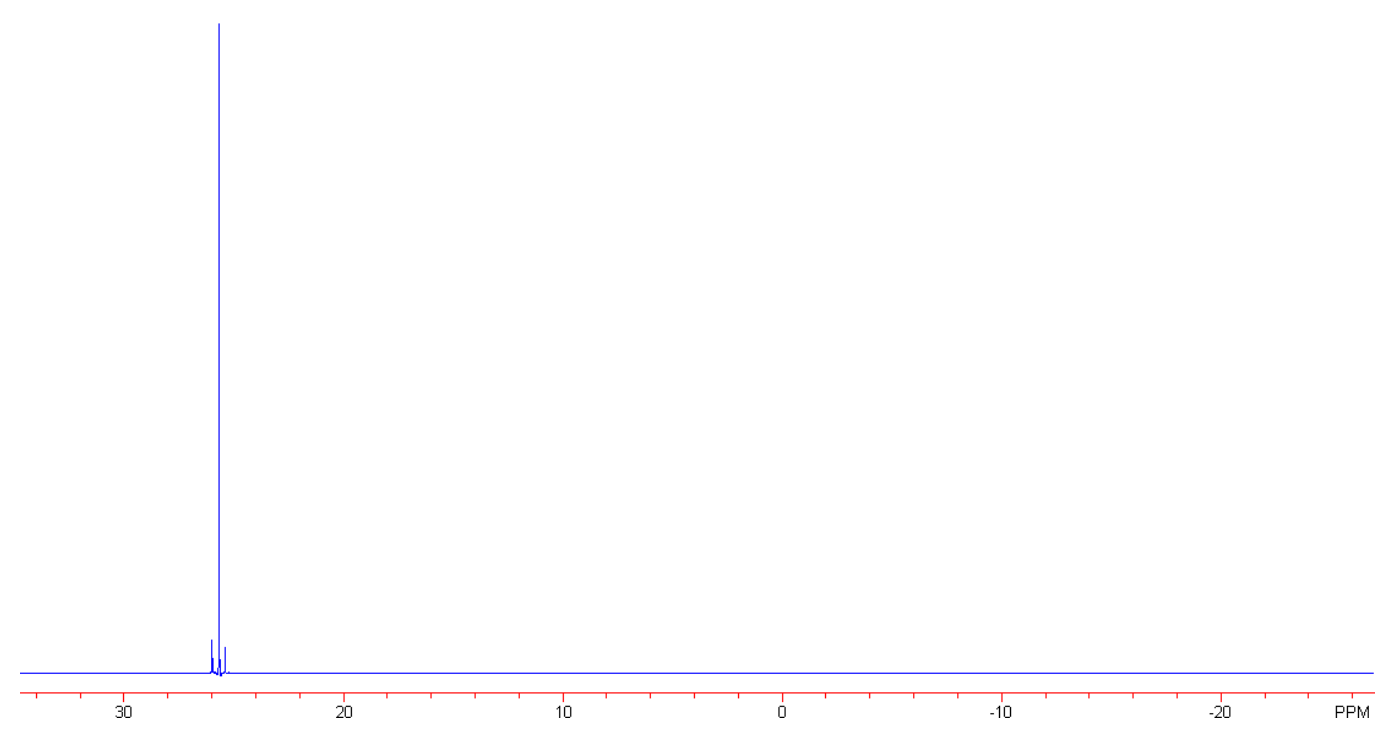

Figure 15. ${ }^{31} \mathrm{P} \mathrm{NMR}$ of meso diastereomer of $\mathrm{DEPN}_{2}\left(162 \mathrm{MHz}, \mathrm{CDCl}_{3}, 25^{\circ} \mathrm{C}\right)$ 


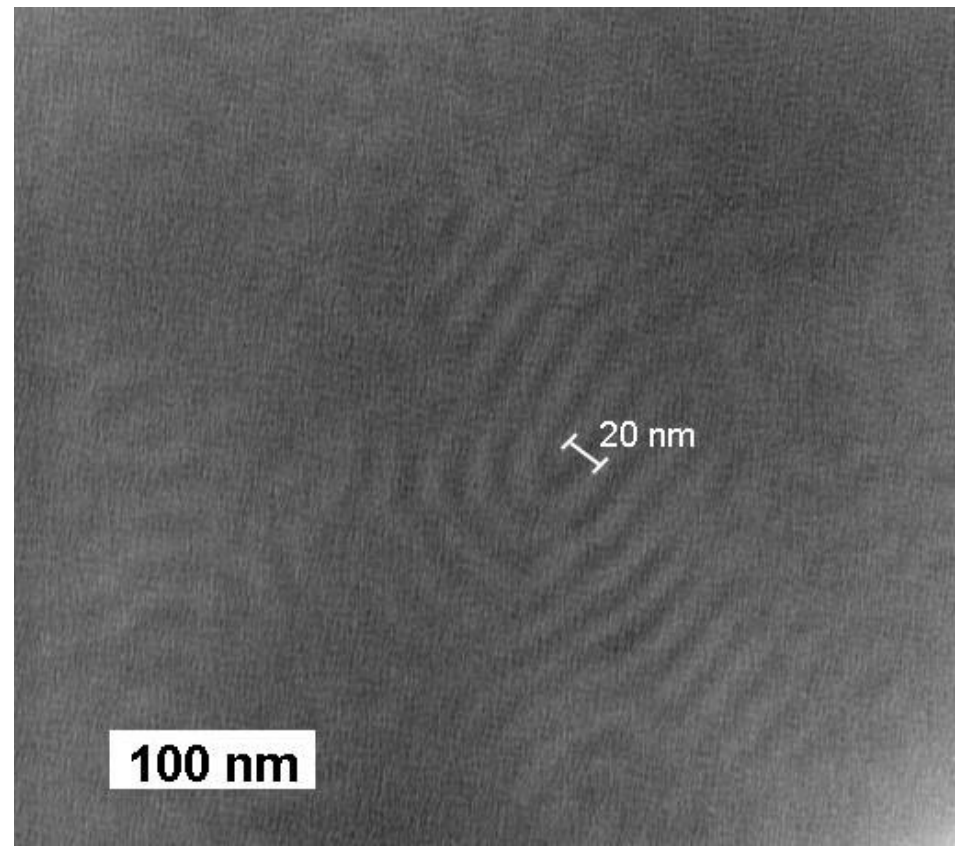

Figure 16. TEM image of blend of $\mathrm{UP}^{+}$and adenine-functional block copolymer (stained with $\mathrm{OsO}_{4}$ ). 
<smiles>C/C=C(\C)c1ccc(Cn2cnc3c(N)ncnc32)cc1</smiles>
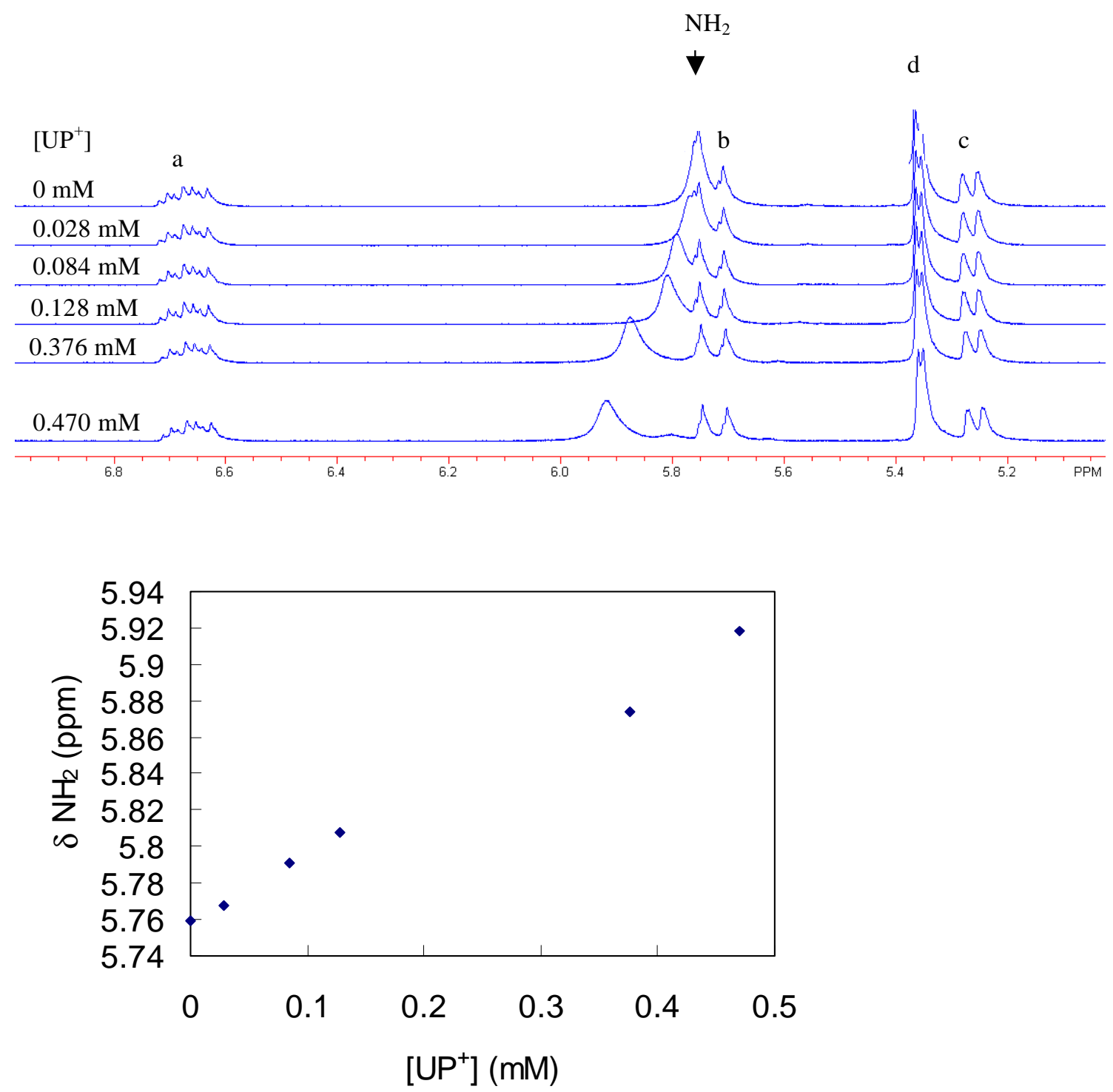

Figure 17. ${ }^{1} \mathrm{H}$ NMR titration of 9-VBA $(1.62 \mathrm{mM})$ with $\mathrm{UP}^{+}$, the molar concentrations of $\mathrm{UP}^{+}$ are listed on the left side of the stacked NMR spectra. 